\title{
Advances in personalized targeted treatment of metastatic melanoma and non-invasive tumor monitoring
}

\author{
Dragana Klinac ${ }^{1}$, Elin S. Gray ${ }^{1}$, Michael Millward ${ }^{2}$ and Mel Ziman ${ }^{1,3}$ \\ 1 School of Medical Sciences, Edith Cowan University, Perth, WA, Australia \\ 2 School of Medicine and Pharmacology, University of Western Australia, Crawley, WA, Australia \\ ${ }^{3}$ School of Pathology and Laboratory Medicine, University of Western Australia, Crawley, WA, Australia
}

Edited by:

Mike Eccles, University of Otago,

New Zealand

\section{Reviewed by:}

Christopher Wong, Genome Institute of Singapore, Singapore

Suzie Chen, Rutgers University, USA

*Correspondence:

Elin S. Gray, School of Medical Sciences, Edith Cowan University, 270 Joondalup Drive, Joondalup,

Perth, WA 6027, Australia.

e-mail: e.gray@ecu.edu.au
Despite extensive scientific progress in the melanoma field, treatment of advanced stage melanoma with chemotherapeutics and biotherapeutics has rarely provided response rates higher than $20 \%$. In the past decade, targeted inhibitors have been developed for metastatic melanoma, leading to the advent of more personalized therapies of genetically characterized tumors. Here we review current melanoma treatments and emerging targeted molecular therapies. In particular we discuss the mutant BRAF inhibitors Vemurafenib and Dabrafenib, which markedly inhibit tumor growth and advance patients' overall survival. However this response is almost inevitably followed by complete tumor relapse due to drug resistance hampering the encouraging initial responses. Several mechanisms of resistance within and outside the MAPK pathway have now been uncovered and have paved the way for clinical trials of combination therapies to try and overcome tumor relapse. It is apparent that personalized treatment management will be required in this new era of targeted treatment. Circulating tumor cells (CTCs) provide an easily accessible means of monitoring patient relapse and several new approaches are available for the molecular characterization of CTCs. Thus CTCs provide a monitoring tool to evaluate treatment efficacy and early detection of drug resistance in real time. We detail here how advances in the molecular analysis of CTCs may provide insight into new avenues of approaching therapeutic options that would benefit personalized melanoma management.

Keywords: metastatic melanoma, personalized treatment, targeted therapy, drug resistance, circulating tumor cells

\section{INTRODUCTION}

Melanoma is an aggressive cutaneous cancer that arises from melanocyte cells within the basal layer of the epidermis. This aggressive malignancy accounts for more than $80 \%$ of skin cancer deaths and its incidence is increasing worldwide (Linos et al., 2009). Cutaneous melanoma arises from the transformation of melanocytes by the accumulation of mutations in genes that regulate cell differentiation and proliferation. The disease manifests itself as clinically and genetically distinct subgroups indicating the need for patient-specific treatment strategies.

In the past decade, since the discovery of key mutations and activated pathways that drive the development of melanoma (Davies et al., 2002), new targeted therapies have been developed, with mixed success. In the fore front of these is a molecule that specifically inhibits the mutated $\mathrm{BRAF}^{\mathrm{V} 600 \mathrm{E}}$ kinase, Vemurafenib, which was approved by the FDA in 2011 as a therapeutic option for treatment of unresectable metastatic melanoma (Chapman et al., 2011). Given the success of this treatment and other treatment advances detailed below, new guidelines for the treatment of melanoma are evolving (Fox et al., 2013). Moreover, deep sequencing analyses have revealed new potential targets and much has been learned about the molecular basis of melanoma genesis. A clearer landscape of the mutation profile of melanoma is emerging and with it new potential therapeutic targets.

\section{MUTATIONS IN MELANOMA}

The most commonly observed recurrent mutations in melanoma reside within the MAPK pathway. The MAPK/Extracellular signalregulated kinase (ERK) signaling pathway is commonly activated in melanoma by mutations in BRAF (in 50\% of melanomas), NRAS (10-20\%), and less frequently in MEK1 and MEK2 ( 8\%) (Davies et al., 2002; Curtin et al., 2005; Murugan et al., 2009; Dutton-Regester and Hayward, 2012). Around $70-95 \%$ of all BRAF mutations are a V600E substitution, with an alternative $\mathrm{V} 600 \mathrm{~K}$ in $5-30 \%$ of the cases. BRAF and NRAS mutations are usually exclusive with a Q61R substitution in $\sim 60 \%$ of NRAS mutated cases (Colombino et al., 2012).

Mutations in upstream tyrosine kinase receptors such as KIT (10\%, mainly in acral and mucosal melanoma), ERBB4 $(\sim 19 \%)$ (Prickett et al., 2009), and FGFR2 ( 10\%) (Gartside et al., 2009), can activate both the MAPK/ERK and the PI3K/AKT pathways.

Activating mutations in the kinases PI3K $(\sim 3 \%)$ and AKT $(\sim 1 \%)$ have also been reported, albeit at lower frequencies (Davies et al., 2002; Omholt et al., 2006). More common are mutations or deletions in the tumor suppressor gene PTEN $(\sim 10-27 \%)$, responsible for the negative regulation of the PI3K/AKT pathway (Paraiso et al., 2011). Mutations in PREX2 (14\%), a negative regulator of PTEN, have been described recently (Berger et al., 2012).

Another tumor suppressor gene commonly altered in melanoma is CDKN2A ( $50 \%)$ which regulates the pRB and p53 
pathways (Flores et al., 1996). Additional driver mutations in TP53 ( 20\%), CDK4 ( 3\%), and RB1 ( 3\%) have also been described, as well as a hot-spot in the adapter protein TRRAP (4\%) (Wei et al., 2011). Furthermore, many mutations have been reported in other components that control cellular proliferation, angiogenesis and apoptosis, including glutamate receptors GRIN2A (33\%) (Wei et al., 2011) and GRM3 (16\%) (Prickett et al., 2011), G-protein GQNA (50\% malignant blue nevi and $46 \%$ of uveal melanomas) (Van Raamsdonk et al., 2010), and the kinases MAP3K5 (9\%) and MAP3K9 (15\%) (Stark et al., 2012). Other genomic aberrations include amplifications in MITF (4\%), CDK4 (3\%), CCND1 (11\%) and TERT (13\%), and deletions in CDKN2A (38\%) (Hodis et al., 2012).

A recent study described five new genes containing potential driver mutations, PPP6C, RAC1, SNX31, TACC1, STK19, and ARID2. The serine/threonine phosphatase PPP6C which negatively regulates the CCND1 oncogene, appears mutated in $12 \%$ of sun-exposed melanomas (Krauthammer et al., 2012), with the R264C substitution in 3\% of cases (Hodis et al., 2012). RAC1, a RAS-related member of the Rho family of GTPases which regulate cytoskeleton rearrangements, contains the P29S substitution in around $4 \%$ of melanomas (Hodis et al., 2012). SKT19, a predicted kinase of known function, contains a D89N mutation in around $5 \%$ of melanomas.

Taken together, these recent tour de force studies reveal the complex array of mutations and genetic aberrations associated with melanoma genesis. Nevertheless it seems apparent that no other single mutation will have the same level of frequency as $\mathrm{BRAF}^{\mathrm{V} 600 \mathrm{E}}$, which is mutated in approximately $50 \%$ of human melanomas (Davies et al., 2002). Further analyses to discern driver from passenger mutations as well as their mechanisms of action are required to clarify the intervention targets and rational combination strategies likely to provide the most successful outcomes. What is abundantly clear, however, is that future therapies will require previous knowledge of the patient's mutational status to guide the most appropriate intervention in a personalized fashion. So far only the targeted inhibitor of BRAF ${ }^{\mathrm{V} 600 \mathrm{E}}$ Vemurafenib has been approved for treatment of melanoma, however we foresee in the near future that an arsenal of therapies will be available based on the tumor genotype. Thus, it is envisaged that tumor specimens will in future, be subjected to targeted sequencing of all the potential mutation hot-spots for which there are therapeutic targets or which affect treatment outcome. However given the inter- and intra-tumor heterogeneity analysis of circulating melanoma cells may provide a comprehensive and sensitive tool for determining the overall mutation status of a patient's tumors.

\section{CLINICAL ADVANCES IN MELANOMA TARGETED THERAPIES BRAF $^{\mathrm{V} 600 \mathrm{E}}$ INHIBITORS}

Developments in molecular targeted therapies (Figure 1; Table 1) have predominantly focused on targeting the BRAF, MEK, or c-KIT kinases located within the MAPK pathway. Two

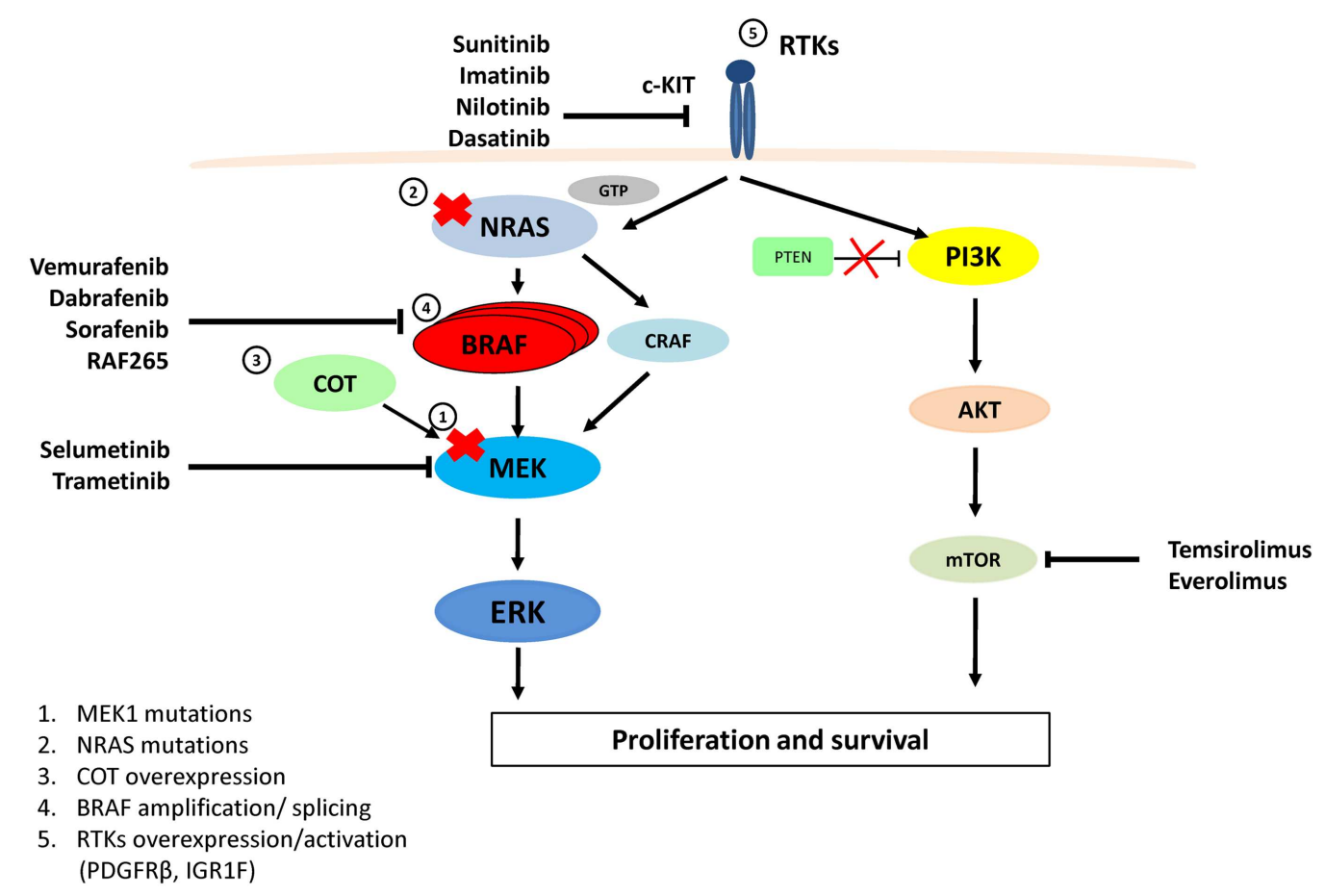

FIGURE 1 | MAPK and PI3K/AKT pathways, therapeutic targets for melanoma and resistance to Vemurafenib. Vemurafenib and Dabrafenib are specific for BRAFV600E, while Sorafenib and RAF-265 are pan-RAF inhibitors. Imatinib, Nilotinib, Dasatinib, and Sunitinib target and inhibit c-KIT. Selumetinib and Trametinib inhibit MEK activity. Temsirolimus and Everolimus inhibit the mTOR protein. Resistance to Vemurafenib arises from MAPK pathway reactivation by (1) a MEK1 $1^{\text {c121s }}$ mutation, (2) NRAS ${ }^{061 R / K}$ mutations, (3) COT1 overexpression, (4) alternatively spliced variants of BRAFVG00E or amplification of the mutant BRAF allele, (5) Overexpression or activation of RTKs (PDGFR $\beta$ or IGF1R) bypasses mutant BRAF and activates ERK via CRAF-MEK or through independent ERK mechanisms by activating the PI3K/AKT pathway. 
Table 1 | Anti-cancer inhibitors undergoing testing for treatment of cutaneous melanoma.

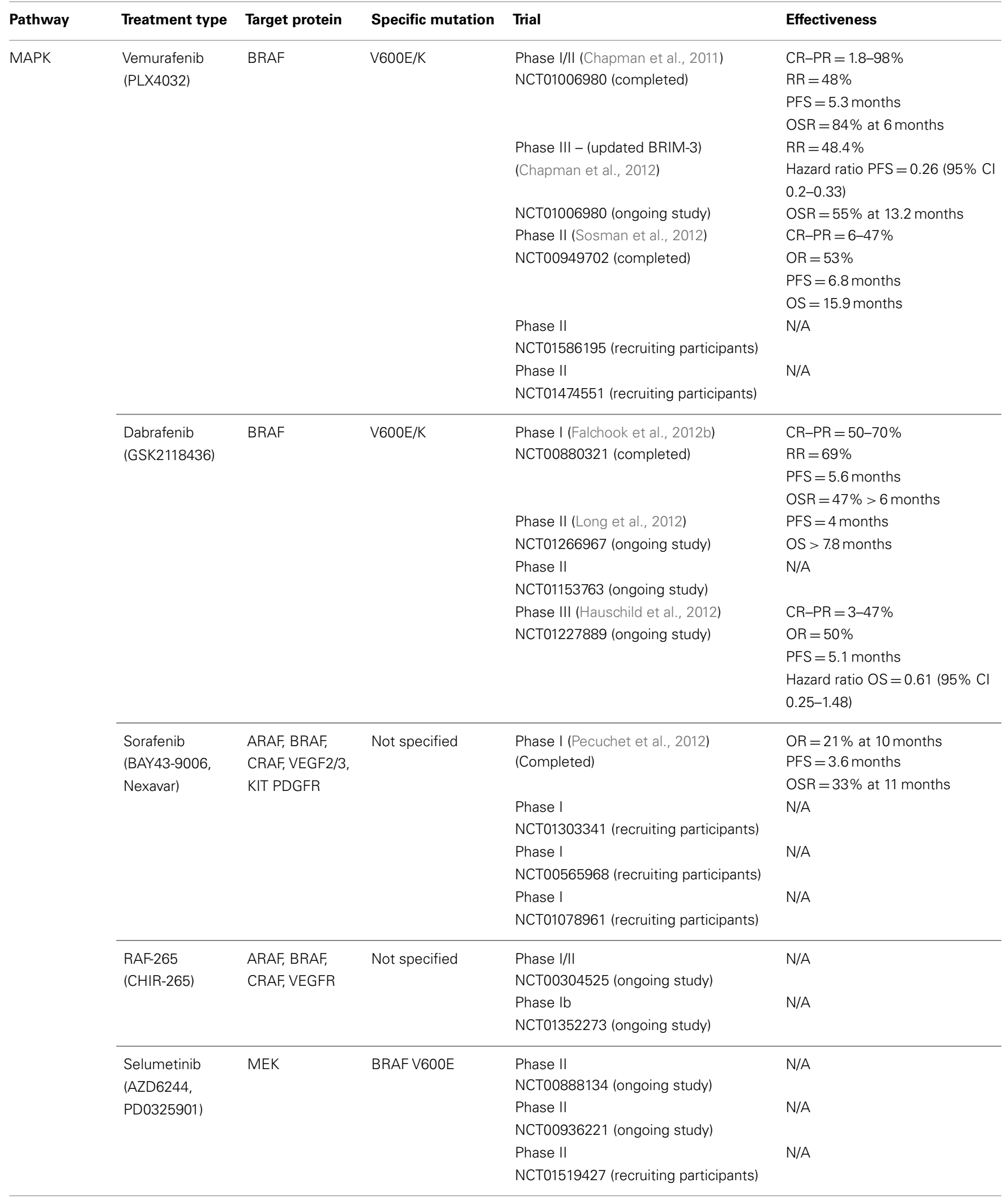


Table 1 | Continued

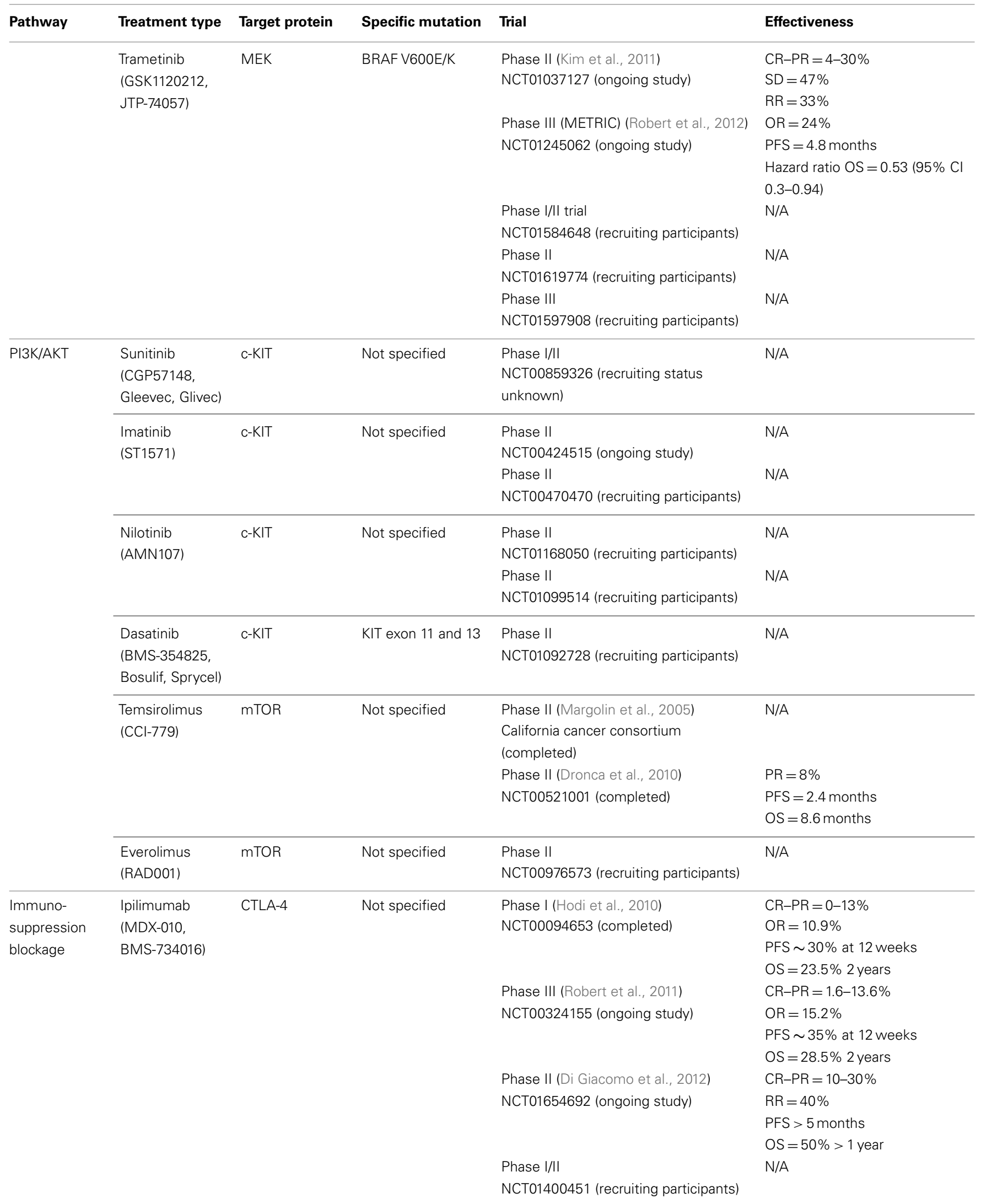


Table 1 | Continued

\begin{tabular}{|c|c|c|c|c|c|}
\hline Pathway & Treatment type & Target protein & Specific mutation & Trial & Effectiveness \\
\hline & \multirow{5}{*}{$\begin{array}{l}\text { MDX-1106 } \\
\text { (BMS-93558 or } \\
\text { ONO-4538) }\end{array}$} & \multirow{5}{*}{ PD-1 } & \multirow{5}{*}{ Not specified } & NCT00730639 (ongoing study) & PFS at 24 weeks $=41 \%$ \\
\hline & & & & NCT01621490 (recruiting participants) & \\
\hline & & & & Phase I & N/A \\
\hline & & & & NCT01176474 (recruiting participants) & \\
\hline & & & & NCT01721772 (recruiting participants) & \\
\hline & \multirow[t]{2}{*}{ MK-3475 } & \multirow[t]{2}{*}{ PD-1 } & \multirow[t]{2}{*}{ Not specified } & Phase I (Hamid, 2012) & $\mathrm{RR}=51 \%$ \\
\hline & & & & NCT01295827 (recruiting participants) & $\mathrm{CR}=9 \%$ \\
\hline & BMS-936559 & PD-L1 & Not specified & Phase I (Brahmer et al., 2012) & $\mathrm{OR}=17 \%$ \\
\hline
\end{tabular}

$P R$, partial response; $R R$, response rate; $C R$, complete response; OR, overall response; PFS, progression-free survival; OS, overall survival; OSR, overall survival rate; $S D$, stable disease; N/A, data not available.

selective BRAF ${ }^{\mathrm{V} 600 \mathrm{E}}$ inhibitors Vemurafenib (commonly known as PLX4032, RG7204, or Zelboraf) and GSK2118436 (Dabrafenib) have demonstrated significant anti-tumor activity (Anforth et al., 2012; Falchook et al., 2012b; Long et al., 2012).

Vemurafenib inhibits the mutant $\mathrm{BRAF}^{\mathrm{V} 600 \mathrm{E}}$ protein and also has inhibitory actions against other kinases, including CRAF, ARAF, and wild-type BRAF (Bollag et al., 2010). The phase III clinical trial (NCT01006980) compared the effect of oral Vemurafenib treatment (960 mg twice daily) to Dacarbazine $\left(1000 \mathrm{mg} / \mathrm{m}^{2}\right.$ intravenous every 3 weeks) in a total of 675 metastatic melanoma patients with the BRAF ${ }^{\mathrm{V} 600 \mathrm{E}}$ mutation. Response rates of more than $48 \%$ were observed in Vemurafenib treated patients compared to a $5 \%$ response rate in the Dacarbazine arm. The estimated median PFS (progression-free survival) for Vemurafenib was 5.3 months with an $84 \%$ overall survival at 6 months, compared to a median PFS of 1.6 months with a $64 \%$ overall survival at 6 months for Dacarbazine (Chapman et al., 2011). As a result of this study, Vemurafenib was approved by the US FDA in August 2011 as a new treatment standard for patients with unresectable or metastatic melanoma with a BRAF ${ }^{\mathrm{V} 600 \mathrm{E}}$ mutation (US Food and Drug Administration, 2011).

A separate phase II clinical trial of Vemurafenib treatment for patients with an activating BRAF ${ }^{\mathrm{V} 600}$ mutation (NCT00949702) included 132 previously treated melanoma patients. Patients were assessed for response rate, duration of the response, and overall survival after Vemurafenib treatment (Sosman et al., 2012). Patients received oral Vemurafenib at a dose of $960 \mathrm{mg}$ twice daily. A complete response was reported in $6 \%(n=8)$ of patients and a partial response was achieved in $47 \%(n=62)$ of individuals with an overall response rate of $53 \%$. Stable disease was noted in $29 \%(n=38)$ of patients, while $14 \%(n=18)$ of subjects demonstrated progressive disease. At the time of data analysis, patients demonstrated a median PFS of 6.8 months and an overall survival of 15.9 months (Sosman et al., 2012).

Common adverse events related to Vemurafenib treatment included fatigue, skin rash, joint pain, photosensitivity, nausea, and development of cutaneous squamous cell carcinomas (SCC) or keratoacanthoma (KA). From the 130 patients that presented adverse reactions to Vemurafenib, 34 patients developed a Grade III SSC or KA. More recently, Su et al. (2012a) reported the paradoxical activation of the MAPK pathway by Vemurafenib; Vemurafenib accelerates the growth of pre-existing cancerous lesions (SSC and KA) via upstream MAPK signaling, such as through

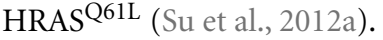

At the 2012 ASCO Annual Meeting, results were reported of the ongoing phase III (BRIM-3) randomized trial (NCT01006980) comparing Vemurafenib with Dacarbazine in previously untreated patients with BRAFV600E melanomas (Chapman et al., 2012). In this trial, a total of 675 patients were randomly assigned to receive either $960 \mathrm{mg}$ of oral Vemurafenib twice daily or Dacarbazine $1000 \mathrm{mg} / \mathrm{m}^{2}$ intravenously every 3 weeks. The median overall survival with Vemurafenib was 13.2 months compared to 9.6 months with Dacarbazine. The 12-month overall survival rates were $55 \%$ for Vemurafenib and $43 \%$ for the Dacarbazine patients. The hazard ratio for death was 0.62 in favor of the Vemurafenib patients. This study confirms the finding that a targeted therapy, Vemurafenib, improves overall survival rates for patients relative to treatment with a chemotherapeutic agent, Dacarbazine (Chapman et al., 2012).

Dabrafenib (GSK2118436) is a reversible, potent ATPcompetitive inhibitor that blocks BRAF ${ }^{\mathrm{V} 600 \mathrm{E}}$ kinase fivefold more effectively that it does CRAF or wild-type BRAF. A phase I dose-escalation trial (NCT00880321) reported active inhibition of melanoma and brain metastases in response to Dabrafenib treatment (Falchook et al., 2012b). A total of 156 patients with metastatic melanoma were involved in the study; 3 of these patients were BRAF wild-type with the other 153 presenting with various $\mathrm{BRAF}^{\mathrm{V} 600}$ mutations. Overall, $47 \%$ of metastatic melanoma patients with a BRAF ${ }^{\mathrm{V} 600 \mathrm{E}}$ mutation maintained successful treatment for more than 6 months. A partial or complete response to Dabrafenib (dosage of $150 \mathrm{mg}$ twice daily) was also noted in $18 \mathrm{BRAF}^{\mathrm{V} 600 \mathrm{~K}}$ mutation positive melanoma patients who were 
given varied doses of Dabrafenib (100-150 mg either once daily or twice daily). Of these, 39\% $(n=7)$ demonstrated a partial response to treatment and $22 \%(n=4)$ had a complete response to treatment. The median PFS for eight patients receiving Dabrafenib $150 \mathrm{mg}$ twice daily was 5.6 months. For three patients with wildtype BRAF, PFS was 1.5 months. The PFS for patients presenting complex BRAF mutations (K601 and V600-K601insdelE) was 1.8 months. For BRAF ${ }^{\mathrm{V} 600 \mathrm{E}}$ patients who did not respond to treatment, PFS was 4.2 month. This study found Dabrafenib to be an effective inhibitor of mutant $\mathrm{BRAF} \mathrm{F}^{\mathrm{V} 600 \mathrm{E} / \mathrm{K}}$ in metastatic melanoma patients with brain metastases and other solid tumors (Falchook et al., 2012b).

A follow-up phase II multicenter trial (NCT01266967) was conducted over six countries, with a total enrollment of 172 metastatic melanoma patients with confirmed $\mathrm{BRAF}^{\mathrm{V} 600 \mathrm{E}}(n=139,81 \%)$ or $\mathrm{BRAF}^{\mathrm{V} 600 \mathrm{~K}}(n=33,19 \%)$ mutations and a brain metastasis. Patients were divided into two cohorts: cohort A consisted of patients who had not received previous treatment for brain metastases and cohort B, subjects had progressive brain metastases after previous treatment. All patients received $150 \mathrm{mg}$ of oral Dabrafenib twice daily. In both $\mathrm{BRAF}^{\mathrm{V} 600 \mathrm{E}}$ and $\mathrm{BRAF}^{\mathrm{V} 600 \mathrm{~K}}$ patients, overall survival was greater than 7.8 months. Interestingly, the overall response was lower amongst patients with a $\mathrm{BRAF}^{\mathrm{V} 600 \mathrm{~K}}$ melanoma than it was in $\mathrm{BRAF}^{\mathrm{V} 600 \mathrm{E}}$ patients. For example, in cohort A intracranial responses were achieved in $39.2 \%(n=29)$ of $\mathrm{BRAF}^{\mathrm{V} 600 \mathrm{E}}$ patients compared to the $6.7 \%$ $(n=1)$ response obtained in $\mathrm{BRAF}^{\mathrm{V} 600 \mathrm{~K}}$ melanomas (Long et al., 2012).

An ongoing phase III randomized controlled trial (NCT01227889) reported recently showed an overall improved PFS for patients with $\mathrm{BRAF}^{\mathrm{V} 600 \mathrm{E}}$ mutant metastatic melanoma treated with Dabrafenib compared with Dacarbazine (Hauschild et al., 2012). A total of 187 patients received Dabrafenib (150 mg twice daily) and 63 patients were given intravenous Dacarbazine ( $1000 \mathrm{mg} / \mathrm{m} 2$ every 3 weeks). The median PFS for the Dabrafenib patients was 5.1 months compared to 2.7 months for the Dacarbazine patients. The complete response rate for the Dabrafenib patients was $3 \%(n=6)$ compared with a $2 \%(n=1)$ response rate for the Dacarbazine group. A $47 \%(n=87)$ partial response rate was reported for the Dabrafenib subjects with a $5 \%(n=3)$ partial response rate observed in the Dacarbazine group. As this clinical study is ongoing, the current overall survival hazard ratio reported is 0.61 (95\% CI 0.25-1.48) in favor of Dabrafenib (Hauschild et al., 2012) but in contrast to the Vemurafenib phase III trial, in this trial all patients randomized to Dacarbazine were given the opportunity to cross over to Dabrafenib on progression masking any overall survival difference. Interestingly, Dabrafenib treatment showed less phototoxic reactions and proliferative epidermal lesions (SCC and KA) in only $6 \%$ of patients, compared to $11 \%$ under Vemurafenib treatment. On the other hand, inflammatory syndromes with fever, rare with Vemurafenib (6\%), were recorded in $20 \%$ of Dabrafenib treated patients (Hauschild et al., 2012; Sosman et al., 2012).

Overall, treatment with Vemurafenib or Dabrafenib confers a survival advantage in metastatic melanoma patients and presents an encouraging treatment option. However, response to these two inhibitors is restricted to only a proportion of melanoma patients.
Efforts to treat metastatic melanoma patients with broad spectrum multi-kinase inhibitors, as detailed below, would seem to be more broadly efficacious since they are independent of BRAF activating mutations, but in fact they are less so.

\section{MULTI-KINASE INHIBITORS}

RAF multi-kinase inhibitor, Sorafenib (BAY 43-9006 or Nexavar), is an oral agent that inhibits many cellular targets including: VEGFR-2, platelet-derived growth factor receptor (PDGFR), cKIT, FLT-3, CRAF, and BRAF. In vitro studies have demonstrated that Sorafenib induces cell cycle arrest and apoptosis in melanoma cell lines via MAPK activity inhibition (Gray-Schopfer et al., 2007). Sorafenib has been granted FDA approval for the treatment of advanced clear-cell renal carcinoma (Wilhelm et al., 2006), based on a randomized trial demonstrating prolonged PFS in patients (Escudier et al., 2007). However, it has demonstrated modest treatment outcomes in patients with advanced melanoma (Eisen et al., 2006; Flaherty, 2006). A recent phase II clinical trial (NCT00119249) confirmed that Sorafenib monotherapy had limited activity in patients with metastatic melanoma regardless of the BRAF ${ }^{\mathrm{V} 600 \mathrm{E}}$ mutational status of their tumor tissue (Ott et al., 2010). By contrast, a more recent study of 28 melanoma patients, showed that after 10 months follow-up there was a $21 \%$ overall response rate with a median PFS of 3.6 months and a 1-year survival rate of $33 \%$ (Pecuchet et al., 2012). Although Sorafenib has not shown increased PFS in melanoma patients there are ongoing clinical trials (NCT01303341, NCT00565968, and NCT01078961) currently recruiting participants which are investigating the effects of Sorafenib in combination with other treatments.

Broad spectrum kinase inhibitors RAF-265 and XL281, known to target ARAF, BRAF, CRAF genes, and VEGFR receptors, have greater effectiveness and modestly improved selectivity for targeting BRAF compared with Sorafenib, in preclinical models and in patients with advanced solid tumors (Venetsanakos et al., 2006; Schwartz et al., 2009). A study using orthotropic implants of metastatic melanoma in mice, showed a $41 \%$ response rate, with more than $50 \%$ reduction in tumor growth after treatment with RAF-265 (Su et al., 2012b). Since the development of more potent BRAF inhibitors, clinical evaluation of RAF-265 inhibitor as a single-agent treatment for melanoma patients is not a strong focus. There is however, an ongoing phase I/II clinical trial (NCT00304525) evaluating the maximum tolerated dose of RAF265 as an oral agent in patients with locally advanced or metastatic melanoma. Another ongoing phase Ib study (NCT01352273) is investigating the combination of the MEK inhibitor (MEK162) with RAF-265 in patients with advanced solid tumors harboring $\mathrm{BRAF}^{\mathrm{V} 600 \mathrm{E}}$ mutations and/or RAS mutations.

It is critical for the field of melanoma therapeutics, to enhance the longevity of the successful responses obtained with BRAF inhibitors. Therefore the focus now is on novel inhibitors designed to target other kinases within the MAPK pathway, for use individually or in combination strategies as additional treatment options.

\section{NRAS INHIBITION}

Inhibition of NRAS has proven challenging as its GTPase activity has not allowed for successful design of specific small-molecule 
antagonists. RNA ( melanoma cell lines (224 and BL, which harbor a Q61R NRAS mutation) inhibits proliferation and renders cells more sensitive to chemotherapy (Eskandarpour et al., 2005). A single-agent, singlearm phase II trial conducted with metastatic melanoma patients investigated Farnesyltransferase inhibitors (FTI's) which block farnesylation, the key activating post-translational modification of RAS (Sebti, 2005). The outcome of this trial using the FTI Tipifarnib (otherwise known as R115777), showed a low response in the first 14 patients which led to early closure of the trial (Gajewski et al., 2006). However, in this trial patients were not selected based on the presence or absence of NRAS mutations.

Due to the absence of successful specific RAS inhibitors for the treatment of melanoma, there are currently no registered clinical trials for the evaluation of NRAS inhibitors. Inhibition of RAS effector pathways would appear to be a more favorable option and investigations of these are underway. The next kinase in the pathway, MEK, has proven to be a more favorable target (Flaherty et al., 2012a).

\section{MEK INHIBITORS}

Selumetinib (also known as AZD6244, ARRY-142886, or PD0325901) is a selective non-ATP-competitive inhibitor of the mitogen-activated protein/ERK kinase (MEK1/2) (Figure 1) (Davies et al., 2007). A successful early phase I trial with Selumetinib, opened the door for MEK inhibitors to be considered as efficacious for patients with metastatic cancer (Lorusso et al., 2005). In this phase I study, the BRAF status of most patients was unknown. However, two cases with known $\mathrm{BRAF}^{\mathrm{V} 600 \mathrm{E}}$ and one with an NRAS (not specified) mutation, displayed a positive response to treatment (Lorusso et al., 2005; Davies et al., 2007). A later phase II single-agent trial compared Selumetinib to Temozolomide. In this study of 100 genetically tested patients, 67 were BRAF and 24 were NRAS positive patients. Only six patients (five of them BRAF positive) receiving Selumetinib showed an $11 \%$ response rate. It is unclear why this trial showed such low response rates in patients and did not show a significant PFS rate relative to Temozolomide (Dummer et al., 2008). However a currently recruiting, phase II clinical trial (NCT01519427) will be investigating the efficacy of a combination of Selumetinib and the AKT inhibitor MK2206, for BRAF positive stage III and/or IV melanoma patients who had previously relapsed whilst on Vemurafenib or Dabrafenib treatment.

MEK162 (also referred to as ARRY-162 or ARRY-438162) is a selective ATP-non-competitive inhibitor of MEK1/2 which inhibits the MEK protein as well as ERK phosphorylation in numerous cancer cell lines (Roberts and Der, 2007; Yeh et al., 2007). The phase I study of orally administered MEK162 in 28 patients with biliary tract cancer showed the drug was well tolerated and had clinical efficacy in patients. An $8 \%(n=2$ of 26 patients) overall response rate was observed in this study population. One patient was reported to have a complete response with a PFS of 8.1 months and another subject had a partial response to treatment with a PFS of 9.8 months. Overall $46 \%(n=12)$ of patients had stable disease outcomes (Finn et al., 2012). Due to the overall positive response to treatment reported in this study, a phase II clinical trial (NCT01320085) investigating the safety and efficacy of MEK162 in patients with advanced or unresectable metastatic malignant melanoma, harboring $\mathrm{BRAF}^{\mathrm{V} 600}$ or NRAS mutations, is currently underway.

Trametinib (known as GSK1120212 or JTP-74057) is a selective oral MEK1/2 inhibitor which mediates blockage of the MAPK kinase MEK protein. Trametinib has been associated with improved PFS and overall survival in patients harboring

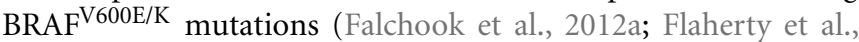
2012b). In a phase II trial (NCT01037127), patients harboring $\mathrm{BRAF}^{\mathrm{V} 600 \mathrm{E} / \mathrm{K}}$ mutant melanoma were given $2 \mathrm{mg}$ of oral Trametinib once daily. Of the patients who were previously treated with BRAF inhibitors $(n=40), 3 \%$ had complete response, $25 \%$ stable disease, and the median PFS was 1.8 months. By contrast, patients who previously received chemotherapy $(n=57), 4 \%$ had complete responses, $30 \%$ had partial responses, and $47 \%$ stable disease. This minimal activity observed in patients previously treated with BRAF inhibitors suggests that BRAF resistant mechanisms may also confer resistance to MEK inhibitor monotherapy (Kim et al., 2011).

Following this trial, an ongoing phase III randomized trial (NCT01245062) was initiated to investigate the efficacy of Trametinib compared to chemotherapy in patients with $\mathrm{BRAF}^{\mathrm{V} 600 \mathrm{E} / \mathrm{K}}$ advanced or metastatic melanoma. Of the 322 enrolled patients, 214 received Trametinib while 108 received chemotherapy. At the time of analysis the confirmed overall response rate was $24 \%$ in the Trametinib patients and 7\% in the chemotherapy group. A median PFS of 4.8 months for the Trametinib patients compared to 1.4 months for the chemotherapy patients was reported. The hazard ratio of overall survival was 0.53 (95\% CI 0.30 $0.94 ; p=0.0181$ ), favoring the Trametinib subjects. Frequent adverse events in the Trametinib patients included skin rash, diarrhea, edema, hypertension, and fatigue. This study found that, compared with chemotherapy, Trametinib provided a significant improvement in progression-free and overall survival for patients with metastatic melanoma (Robert et al., 2012).

\section{COMBINATION TARGETED THERAPIES}

More recently, greater improvements have been noted in metastatic melanoma patients treated with combination targeted therapies, particularity so the combination of BRAF (Dabrafenib) and MEK (Trametinib) inhibitors (Flaherty et al., 2012a). When used as a single-agent Dabrafenib, like Vemurafenib, has shown patients developing resistance after approximately 5-7 months (Falchook et al., 2012b; Hauschild et al., 2012; Long et al., 2012). Therefore the rationale for adding a MEK inhibitor is that it may block the escape route for the BRAF inhibitor and allow continual response and remission in patients. The phase I clinical trial NCT01072175 tested the combination of oral Dabrafenib (150 mg) and Trametinib (2 mg) compared to Dabrafenib $(150 \mathrm{mg})$ alone in 162 patients with metastatic melanoma containing the BRAF ${ }^{\mathrm{V} 600 \mathrm{E} / \mathrm{K}}$ mutation. The Dabrafenib group $(n=54)$ had a median PFS of 5.8 months compared with a 9.4 month PFS in the combination group $(n=54)$ (Flaherty et al., 2012a). Currently the phase III trial (NCT01682083) is underway in metastatic $\mathrm{BRAF}^{\mathrm{V} 600}$ mutated melanoma patients comparing treatment combinations of Dabrafenib and Trametinib versus Dabrafenib alone. Other clinical trials currently recruiting 
patients with advanced or metastatic melanoma using the combination of Trametinib and Dabrafenib include NCT01619774, NCT01584648, NCT 01072175, and NCT01597908.

\section{KIT INHIBITORS}

Activating mutations in c-KIT result in stimulation of the MAPK and PI3K-Akt pathways causing increased proliferation and survival advantages (Figure 1) (Webster et al., 2006). The c-KIT inhibitor Sunitinib is a potent inhibitor of mutant KIT with additional inhibitory effects on VEGF receptors (Chow and Eckhardt, 2007). A recent study conducted by Minor et al. showed that Sunitinib may provide a treatment option for melanoma patients with KIT mutations. Tumor tissues from 90 patients with stage III or IV acral, mucosal or cumulative sun-damaged melanoma were collected. The tumor tissues were sequenced for KIT, BRAF, NRAS, and GNAQ mutant genes and patients with amplification or overexpression of KIT were treated with Sunitinib. Of the melanoma patients treated with Sunitinib, 11\% had mutations in KIT [other patients presented with mutations in BRAF (23\%), NRAS (14\%), or GNAQ (0\%)]. Patients positive for KIT mutations $(n=4$; exon 11; W557G, V559G, or L576P) showed varied responses to the treatment. One patient had complete remission for 15 months, while two patients demonstrated partial responses for 1 and 7 months respectively (Minor et al., 2012). A clinical trial (NCT00859326) is now in progress investigating the efficacy of a combination of Sunitinib and Temozolomide (an oral, cytotoxic chemotherapy agent) for the treatment of metastatic and unresectable malignant melanoma patients.

Imatinib or Imatinib mesylate (also known as ST1571, Gleevec, or Glivec) is a receptor protein kinase inhibitor targeting Abl, cKIT, and the PDGFR (Fecher et al., 2007; Stuart and Sellers, 2009). In two phase II trials in patients with metastatic melanoma, Imatinib has shown no response and poor survival outcomes in 16 and 25 patients, respectively (Ugurel et al., 2005; Wyman et al., 2006). In contrast, a case report revealed that Imatinib may be an effective treatment, since in one patient with a c-KIT mutation in exon 11 , a positive outcome to the treatment was observed (Hodi et al., 2008).

More recently the Imatinib inhibitor has been evaluated as a treatment option in melanoma patients presenting c-KIT mutations (Carvajal et al., 2011; Guo et al., 2011). The phase II trial, in 46 metastatic melanoma patients with c-KIT mutations or amplifications, demonstrated an overall response rate of $23.3 \%$. All patients received a continuous dose of $400 \mathrm{mg}$ of Imatinib, unless toxicities or disease progression occurred. Fifteen patients who experienced reoccurrence were given an increased dose of $800 \mathrm{mg}$ per day. The median PFS for the 46 patients was 3.5 months, with a 6-month PFS rate of $36.6 \%$, and an overall 1-year survival rate of $51 \%$. The overall rate of disease control was $53.5 \%$. This study found that Imatinib increased the overall PFS rate, response rate, and overall survival rate in patients presenting c-KIT mutations in exon 11 and 13. However, patients who had increased doses of Imatinib did not show improvements in disease control (Guo et al., 2011). Ongoing, is the phase II clinical trial (NCT00470470), investigating Imatinib in patients with unresectable stage III or IV melanoma harboring somatic alterations of c-KIT.
Nilotinib (also known as AMN107) is a second generation tyrosine kinase inhibitor known to inhibit KIT, PDGFR, and Bcr-Abl. It was approved by the FDA in 2010 for the treatment of chronic myeloid leukemia $(\mathrm{CML})$ and has a similar target profile to Imatinib (Manley et al., 2010). A phase I clinical trial demonstrated that Nilotinib activity is safe and effective in CML resistant to treatment with Imatinib (Kantarjian et al., 2006) and a major clinical response was observed to Imatinib in KIT-mutated metastatic rectal melanoma (Hodi et al., 2008). A current clinical trial (NCT01168050) is examining Nilotinib as a first or second line treatment of primary melanoma, stage III unresectable, or stage IV melanomas with c-KIT mutations or amplifications (NILOMEL). Another clinical trial (NCT01099514) will also be investigating Nilotinib in metastatic melanoma with KIT aberrations.

Dasatinib (also known as Bosulif, Sprycel, or BMS-354825) is a tyrosine kinase inhibitor responsible for inhibiting src family kinases (c-src, yes, lck, and fyn), Bcr-Abl, c-KIT, PDGF $\beta$ receptor, and EPHA2 (Lombardo et al., 2004). Dasatinib was approved by the FDA for CML and gastrointestinal stromal tumors (GIST) (von Mehren, 2006; Pavlu and Marin, 2009). A recent single-arm phase II study of Dasatinib recruited 17 patients with advanced melanoma. The objective response rate was $5 \%$ with evidence of tumor regression after only four cycles of therapy $(n=5)$. The median PFS was 8 weeks. This study revealed that Dasatinib had limited activity in patients with advanced or unresectable melanoma and did not meet the pre-specified response rate $(30 \%)$ or the 6-month PFS (Kluger et al., 2011). However a clinical trial (NCT01092728) is currently recruiting participants to investigate Dasatinib monotherapy in patients with acral lentiginous mucosal or chronic sun-damaged cutaneous melanoma.

\section{mTOR INHIBITORS}

The therapeutic value of targeting the PI3K/AKT pathway in melanoma has not been as clearly elucidated as it has been for the MAPK pathway. However, it is clear that an active crosstalk between these two pathways supports the development of melanoma and leads to resistance to BRAF inhibitors. Due to the lack of PI3K and AKT inhibitors currently available for clinical trial evaluations in melanoma, attention has turned to mTOR for which several inhibitors are under development.

Temsirolimus, an mTOR inhibitor (also known as CCI-779), is an analog of Sirolimus (rapamycin) that has demonstrated immunosuppressive activity against melanoma in preclinical models and revealed benefits in patients with breast and renal carcinoma (Hidalgo and Rowinsky, 2000; Huang and Houghton, 2003; Lu et al., 2003). By contrast, an early study demonstrated that Temsirolimus activity resulted in poor clinical responses and limited disease PFS rates in metastatic melanoma patients (Margolin et al., 2005).

While this mTOR inhibitor study diminishes the therapeutic value of targeting the PI3K pathway in melanoma, preclinical evidence has shown, however, that co-targeting this pathway along with the MAPK pathway remains an important therapeutic option (Meier et al., 2007). For example, both PI3K and mTOR inhibitors have revealed synergistic responses when used in combination therapies with Sorafenib or MEK inhibitors (Molhoek et al., 
2005; Meier et al., 2007; Lasithiotakis et al., 2008; Chappell et al., 2011). Interestingly the same response has not been generated with BRAF inhibitors (Meier et al., 2005; Molhoek et al., 2005). Current phase I/II clinical trials (NCT00281957, NCT01614301, and NCT01565837) investigating combination treatments which include Sorafenib, MEK inhibitors, chemotherapy agents, and stereotactic ablative radiation therapy along with Temsirolimus in patients with metastatic melanoma or advanced cancers are underway.

Another mTOR inhibitor Everolimus (also known as RAD001) is currently being investigated in patients with metastatic melanoma in the clinical trial NCT00976573, in which the chemotherapeutic agents (Carboplatin and Paclitaxel) and Bevacizumab are used with Everolimus. Another phase II study (NCT00521001) investigated the combination of Everolimus (10 mg daily, for 5 of 7 days) and Temozolomide $\left(200 \mathrm{mg} / \mathrm{m}^{2} 1-\right.$ 5 days, every 28 days) in patients $(n=48)$ with stage IV metastatic melanoma. From the 48 patients, $8 \%(n=4)$ achieved a partial response, the median PFS was 2.4 months and the overall survival was 8.6 months. The combination of Everolimus and Temozolomide did not offer a therapeutic advantage over Temozolomide alone (Dronca et al., 2010). However, a recent phase I study investigating the combination of Everolimus with Capecitabine in patients with advanced solid malignancies demonstrated a prolonged clinical benefit for 39\% of patients (Deenen et al., 2012). Currently two clinical trials (NCT01252251 and NCT00976573) are investigating the therapeutic benefit of Everolimus treatment plus chemotherapy in patients with melanoma.

From the studies detailed above, it is clear that current and future clinical trials will focus on implementing several combination targeted therapies for melanoma patients in the hope of increasing survival rates and minimizing tumor regression. Since improved survival rates have been demonstrated in patients with advanced melanoma, particularly for Vemurafenib and Dabrafenib, trials are underway to develop novel inhibitors that target several genes within the MAPK pathways, as these can be used in combination targeted therapies with the hope of prolonging PFS. However this strategy is for patients with BRAF/NRAS/MEK mutations only. For patients with mutations in alternate pathways (PI3K and AKT) alternate therapies are required. The lack of efficacy to date, when alternate pathways are targeted may imply that combination treatments that also target the MAPK pathways, such as BRAF or MEK inhibitors together with an MTOR inhibitor, are required to prolong PFS and to prevent escape mutations. An alternate therapeutic option is immunotherapy, which is proving to be efficacious (Wilmott et al., 2012).

\section{IMMUNOTHERAPY THERAPIES}

Ipilimumab (also known as Yervoy, MDX-010, or BMS-734016) a monoclonal antibody to the T-lymphocyte associated antigen 4 (CTLA-4) was approved by the US FDA in March 2011 and it is currently implemented as a treatment option for patients with stage III and IV metastatic melanoma. CTLA-4 is member of the immunoglobulin receptor family essential for the development of regulatory T-cells. Signaling through this molecule induces an inhibitory response that abrogates the cytotoxic response of the T-cells. Blocking this inhibitory signaling allows the tumor infiltrating lymphocytes to attack the tumors cells.

A phase III clinical trial (NCT00094653) reported by Hodi et al. (2010) demonstrated an improved survival rate in patients with unresectable stage III and IV melanoma. These patients received Ipilimumab either alone $(n=102)$ or in combination with the glycoprotein 100 peptide vaccine (gp100) $(n=403)$ (Hodi et al., 2010). In another phase III trial (NCT00324155) investigating Ipilimumab in combination with Dacarbazine for patients with previously untreated metastatic melanoma overall survival rates were $47.3 \%$ for 1 -year, $28.5 \%$ for 2 -years, and $20.8 \%$ for 3 -years (Robert et al., 2011). This study demonstrated a slight improvement in the overall survival responses for patients who received Ipilimumab-plus Dacarbazine compared with patients who had received Ipilimumab-plus the gp100 vaccine. Di Giacomo et al. (2012) have reported on a more recent phase II clinical trial (NCT01654692) which assessed the combination of Ipilimumab and Fotemustine in patients with advanced, unresectable stage III or IV melanoma. A total of $46.5 \%(n=40)$ of the study population maintained a stable disease within 12 months and a median PFS of 5 months (Di Giacomo et al., 2012). More than $50 \%$ $(n=10)$ of patients with brain metastases survived longer than 12 months, compared to approximately $20 \%$ survival reported for patients undergoing radiotherapy or surgery (Eigentler et al., 2011). Currently a phase I/II clinical trial (NCT01400451) combining BRAF targeted therapy (Vemurafenib) with immunotherapy (Ipilimumab) is underway in subjects with $\mathrm{BRAF}^{\mathrm{V} 600 \mathrm{E} / \mathrm{K}}$ metastatic melanoma as a strategy to prolong PFS.

Other immunotherapeutic agents currently being tested are antibodies that interfere with the PD-1 (programed death-1) and PD-L1 (PD-1 ligand). PD-1 is a key immune co-inhibitory receptor expressed by activated T-cells which mediate immunosuppression. The primary function of PD-1 is in peripheral tissue where T-cells encounter immunosuppressive ligands PD-L1 (also known as B7-H1 or CD274) and PD-L2 (also referred to as B7DC or CD273) which are expressed by tumor and/or stromal cells (Dong et al., 1999; Menzies et al., 2012; Topalian et al., 2012). Anti-PD-1 antibodies interfere with the interactions between PD1 and PD-L1 allowing the T-cells to attack the tumor cells (Iwai et al., 2002; Dong et al., 2003). The anti-PD-1 inhibitor monoclonal antibody MDX-1106 (also referred to as BMS-936558 and ONO-4538) showed favorable preliminary evidence when administrated as a single-agent in a pilot study involving 39 patients with advanced solid tumors (Brahmer et al., 2010). In another study amongst participants with melanoma $(n=94), 28 \%(n=26)$ had objective responses, lasting for 1 year or more (Topalian et al., 2012). Various trials are underway comparing the clinical benefit and overall survival after treatment with this anti-PD-1 antibody (NCT01621490, NCT01176474, and NCT01721772). MK-3475 is another anti-PD-1 inhibitor which is being investigated in a phase I clinical trial (NCT01295827). Encouraging anti-tumor activity was reported at the recent Society for Melanoma Research Congress in November 2012 (Hamid, 2012). Objective anti-tumor responses were recorded in $51 \%(n=43)$ of 85 patients analyzed to date. Of those $9 \%(n=8)$ of patients demonstrated a complete response to MK-3475. Furthermore, a study conducted by Brahmer et al. (2012) has shown that the anti-PD-L1 antibody 
BMS-936559 provides durable tumor responses in patients with advanced cancer including melanoma. These results validate the interaction between PD-1 and PD-L1 as an important target for therapeutic intervention in melanoma patients.

In general the anti-PD-1 and anti-PD-L1 treatments have achieved the highest rate of anti-tumor activity reported for an immunotherapeutic agent in the past 30 years (Ribas, 2012). Together with Ipilimumab, these immunotherapeutic agents have demonstrated an increased durability of the tumor response (Hodi et al., 2010; Brahmer et al., 2012; Topalian et al., 2012). Their low response rate compared to targeted therapies such as the $\mathrm{BRAF}^{\mathrm{V} 600 \mathrm{E}}$ inhibitors support their use in combination therapies. With two different modes of action, combination therapies that together target both cellular proliferation and immune response might provide enhanced inhibition of the spread of melanoma, and may overcome the development of drug resistance.

\section{RESISTANCE TO BRAF INHIBITORS AND COMBINATION THERAPIES}

Although there have been encouraging results with targeted BRAF inhibitors, such as Vemurafenib and Dabrafenib (Hauschild et al., 2012; Sosman et al., 2012), almost all patients on these therapies develop drug resistance after the initial response, leading to clinical relapse. The underlying reasons for the development of drug resistance can be found in the redundancy of molecular and cellular processes that mediate the development of melanoma (Figure 1). Significant efforts have been dedicated to the study of acquired resistance to BRAF inhibitors. Results from various groups indicate that resistance to BRAF inhibition can be attributed to a series of heterogeneous mechanisms that lead to the reactivation of the MAPK pathway. These mechanisms of reactivation include upregulation of NRAS through activating mutations (Q61K/R) (Nazarian et al., 2010), overexpression of COT/Tp12 by increased copy number of the MAP3K8 locus (Johannessen et al., 2010), activation of MEK1 by mutation C121S (Wagle et al., 2011), alternative BRAF splicing (Poulikakos et al., 2011), or BRAFV600E gene amplification (Shi et al., 2012b). Alternative, resistance is achieved by the activation of PI3K-AKT and RAS-CRAF-MEK pathways through receptor tyrosine kinase (RTK) signaling. Such activation includes, overexpression of platelet-derived growth factor $\beta$ (PDGF $\beta$ ) (Nazarian et al., 2010; Shi et al., 2012b) and activation of IGF1R (Villanueva et al., 2010). Interestingly, all these escape mechanisms are largely mutually exclusive and differ between patients and in some cases between tumors within a patient (Nazarian et al., 2010; Shi et al., 2012b).

\section{MEK MUTATION}

Wagle et al. (2011) profiled tumors sensitive and resistant to BRAF inhibitors by massive parallel sequencing and identified the reactivation of the MAPK pathway by a newly identified mutation, MEK $1^{\mathrm{C} 121 \mathrm{~S}}$. MEK $1^{\mathrm{C} 121 \mathrm{~S}}$ also confers cross-resistance to the MEK inhibitor Selumetinib. However, this mutation has not been observed in any other studies of Vemurafenib-resistant tumors since then (Shi et al., 2012b). On the other hand, commonly found MEK exon 3 activating mutations such as $\mathrm{MEK}^{\mathrm{P} 124 \mathrm{~S}}$ and $\mathrm{MEK}^{\mathrm{I} 111 \mathrm{~S}}$ are shown to not confer Vemurafenib resistance (Shi et al., 2012a). Escape through a MEK activating mutation is therefore unusual and in contrast to most other mechanisms of acquired drug resistance, where the activation emerges downstream of the targeted kinase (Wagle et al., 2011).

\section{NRAS MUTATIONS}

Nazarian et al. demonstrated that acquired resistance to Vemurafenib developed in melanoma cell lines and patient tumors by the acquisition of NRAS mutations. Interestingly, two biopsies from the same patient had two different activating NRAS mutations (NRAS ${ }^{\mathrm{Q} 61 \mathrm{R}}$ and NRAS ${ }^{\mathrm{Q} 61 \mathrm{~K}}$ ) (Nazarian et al., 2010). More recently Shi et al. (2012b) reported that 5 of 15 patients with disease progression after responding to Vemurafenib, carried NRAS mutations. The NRAS mutated cells were sensitive to the MEK inhibitor, Selumetinib, in the presence or absence of Vemurafenib, suggesting that reactivation of the MAPK pathway might have occurred via CRAF bypassing the BRAF inhibition. This was later confirmed by re-sensitization of a cell line $\left(\mathrm{NRAS}^{\mathrm{Q} 61 \mathrm{~K}} / \mathrm{BRAF}^{\mathrm{V} 600 \mathrm{E}}\right.$ ) to Vemurafenib by knocking down CRAF expression (Shi et al., 2012b).

\section{COT OVEREXPRESSION}

Through the screening of an "open reading frame" expression library encoding approximately $75 \%$ of the human kinases, Johannessen et al. (2010) identified that overexpression of COT/Tpl2 and CRAF reduced sensitivity to BRAF inhibitor PLX4720 (a preclinical version of Vemurafenib). Moreover, increased COT transcript levels were observed in two biopsies collected during Vemurafenib treatment and compared to lesion-matched pretreatment biopsies. Furthermore, high levels of COT expression were related to an increased copy number of the MAP3K8 locus in two cell lines resistant to PLX4720. Over-activation of MEK in the melanoma cell line A375 through COT signaling resulted in resistance to the MEK inhibitors Selumetinib and CI-1040. Nevertheless, the authors found that co-inhibition of both BRAF and MEK can overcome resistance to BRAF inhibitors caused by increased COT levels.

\section{REACTIVATION OF BRAF}

Contrary to intuition, no compensatory BRAF mutations have been found as a mechanism of resistance to BRAF inhibitors. However, reactivation of tumor progression after response to BRAF inhibitors can be achieved by tumor cells with an increased copy number of BRAF ${ }^{\mathrm{V} 600 \mathrm{E}}$. Indeed, Shi et al. (2012b) demonstrated that $20 \%$ of melanoma patients treated with BRAF inhibitors (Vemurafenib and Dabrafenib) showed an increase in genomic copy number of $\mathrm{BRAF}^{\mathrm{V} 600 \mathrm{E}}$ and $\mathrm{BRAF}^{\mathrm{V} 600 \mathrm{E}}$ amplification resulted in $\mathrm{BRAF}^{\mathrm{V} 600 \mathrm{E}}$ overexpression in tumors of melanoma patients whose cancer had progressed after initial response. Cell lines with $\mathrm{BRAF}^{\mathrm{V} 600 \mathrm{E}}$ gene amplification, thus resistant to BRAF inhibitors, remained sensitive to Selumetinib, with Vemurafenib and Selumetinib combination therapy producing a synergistic effect.

Poulikakos et al. identified BRAF ${ }^{\mathrm{V} 600 \mathrm{E}}$ splicing variants which lack a RAS-binding domain (RBD) in two cell lines. These cell lines displayed acquired resistance to Vemurafenib, that could not be explained by mechanisms previously described (Poulikakos et al., 2011). The observed truncated form of BRAF (p61BRAF) was 
the result of an in-frame deletion of exons 4-8. While the mechanism underlying this exon skipping phenomena remains to be identified, exons 4-8 encode domains essential for RAF activation, including the RBD and the cysteine-rich domain (CRD) (Wellbrock et al., 2004). The truncated BRAF lacking the RBD is able to dimerize independently of RAS signaling. Introduction of a mutation that abolishes p61BRAF dimerization restored sensitivity to Vemurafenib. Confirming this as a mechanism of resistance, BRAF variants lacking the RBDs were found in 6 of the 19 patients undergoing Vemurafenib treatment (Poulikakos et al., 2011), while Shi et al. (2012b) reported the same mechanism in another two patients. P61BRAF ${ }^{\mathrm{V} 600 \mathrm{E}}$ expressing cells remained sensitive to the MEK inhibitor Selumetinib. It is possible that these mechanisms of resistance may benefit from dose-escalation of the BRAF inhibitor, such as Dabrafenib, for which, the maximum tolerated dose has not yet been determined.

\section{RTK ACTIVATION}

In addition to the above mechanisms of acquired resistance to BRAF inhibitors, RTK overexpression or activation has been shown to bypass mutant BRAF and reactivate ERK through CRAF-MEK or via ERK independent mechanisms by activating the PI3K/AKT pathways. Upregulation of PDGFR $\beta$ and EGFR were demonstrated to mediate resistance to Vemurafenib developed in melanoma cell lines by Nazarian et al. (2010). In particular PDGFR $\beta$ displayed increased activation associated with tyrosine phosphorylation. Moreover the authors found that 4 of 11 post-relapse biopsies from melanoma patients treated with Vemurafenib showed increased expression of PDGFR $\beta$ in comparison to pre-treatment biopsies. The same increase was also observed in three relapse tumor biopsies from a patient treated with Dabrafenib (Shi et al., 2012b).

Platelet-derived growth factor receptor $\beta$ knockdown by RNAi in resistant cell lines led to re-sensitization of the growth inhibition by Vemurafenib, but did not activate the apoptotic response (Nazarian et al., 2010). Thus, PDGFR $\beta$ overexpression might not be the only mechanism of resistance in these cells. Moreover, the PDGFR $\beta$ inhibitor Imatinib or the MEK inhibitor Selumetinib did not restore sensitivity to Vemurafenib (Shi et al., 2011). It is possible that resistance may involve the activation of more than one RTK.

BRAF inhibitor resistance also has been demonstrated to occur via phospho-activation of the RTK, IGF1R, with subsequent downstream activation of the PI3K/AKT pathways (Villanueva et al., 2010). Inhibition of IGF1R led to slower cell survival, but little improvement was observed when added in combination with the BRAF inhibitor. IGFR inhibition diminished pAKT activation, but did not suppress pMEK/pERK activation. Combination IGF1R inhibitor, PPP, with a MEK inhibitor, Trametinib, led to increased apoptosis and decreased cell viability (Villanueva et al., 2010).

Two recent reports showed RTK-mediated resistance to BRAF inhibition in colorectal carcinoma (Corcoran et al., 2012; Prahallad et al., 2012). Both studies showed activation of EGFR and downstream pathways (PI3K/AKT and MEK/ERK). All these studies underscore the role of RTK expression and activation in BRAF inhibitor acquired resistance. Given the redundancy and promiscuity of the RTKs signaling in melanoma cells, RTK reprograming might not effectively halt tumor growth. This leads to a proposition that co-targeting MEK, and the PIK/AKT/mTOR pathway would be a more effective strategy in response to this type of BRAF inhibitor induced resistance (Lo, 2012).

\section{MOLECULAR CHARACTERIZATION OF CIRCULATING TUMOR CELLS FOR PERSONALIZED TREATMENT MONITORING}

Targeted cancer therapies are effective in only a proportion of patients. For effective therapy accurate molecular analysis of a patient's tumors is required, as incorrect administration can negatively impact on patient survival. Molecular tools are required that determine which patients are likely to benefit from the therapy and reveal, early during treatment, whether the therapy is effective. The quantification and molecular profiling of circulating tumor cells (CTCs) has been proposed as an aiding methodology for tumor genotyping and for early detection of therapy efficacy.

Several studies have investigated the value of detecting CTCs in melanoma patients by multimarker RT-PCR to predict response to therapeutic regimens with mixed outcomes. Reynolds et al. (2003) observed that therapy with a polyvalent melanoma vaccine was associated with clearance of melanoma cell markers (tyrosinase, gp100, MART-1, and MAGE-3) from the circulation and improved prognosis. Monitoring of CTCs by expression of five melanoma-associated biomarkers (MART-1, GalNAc-T, PAX3, MAGE-A3, and MITF) in patients receiving biochemotherapy and maintenance biotherapy for stage IV melanoma suggests that CTCs detection may be useful for predicting therapeutic efficacy and disease outcome (Koyanagi et al., 2010; Reid et al., 2013). In a multivariate analysis, pre-treatment and serial CTC positivity (MART-1, MAGE-A3, and PAX-3 RT-PCR) was significantly associated with disease-free survival and overall survival (Hoshimoto et al., 2012) (NCT00052156). However, Fusi et al. (2012) reported that although CTCs positivity (Mart-1 and tyrosinase) was time dependant prognostic factor, it was not predictive of treatment outcome. Overall, CTC quantification using RT-PCR has been deemed prone to false positive results and the lack of validated and standardized methodologies has preclude its use as a biomarker in clinical trials (Nezos et al., 2009).

Several methodologies have been developed for cytometric detection of CTCs. At the fore front of these is the CellSearch system. Using this platform CTCs have been detected in cancer patients at both early and late stages, with the number of tumor cells in peripheral blood showing significant utility for prognosis in breast, colorectal, prostate, and non-small-cell lung cancers (Cristofanilli et al., 2004; Cohen et al., 2008; de Bono et al., 2008; Krebs et al., 2011). More recently, Khoja et al. demonstrated that CTCs were detectable in $40 \%$ of patients with advance cutaneous melanoma and the number of CTCs was prognostic for overall survival. They also showed preliminary evidence that changes in the number of CTCs during treatment may reflect outcome (Khoja et al., 2012). Currently additional trials are underway investigating the prognostic and predictive value of CTCs to identify responding patients treated with Ipilimumab (NCT01565837), Imatinib (NCT00470470), Everolimus (NCT00976573), and BRAFV600E inhibitors (NCT01573494). 
Circulating tumor cells not only constitute seeds for metastases and indicate the spread of the disease, but they also reflect the tumors within a patient, thus genetic changes in tumors could be readily detected in CTCs. Thus, CTCs could constitute an accessible sample with which to analyze the genetic profile of the tumors in a particular individual and possibly better represent the mutation status of all the tumors within a patient than a single biopsy. The detection of the BRAF ${ }^{\mathrm{V} 600 \mathrm{E}}$ mutation in CTCs isolated from melanoma patients has been previously reported (Kitago et al., 2009; Freeman et al., 2012). A recent report by Sakaizawa et al. (2012) successfully identified BRAF and KIT activating mutations at a single cell level in CTCs from patients with melanoma. Another study also showed the detection of BRAF ${ }^{\mathrm{V} 600 \mathrm{E}}$ in CTCs with a $91 \%(19 / 21)$ correspondence with the matched tumor tissue. Moreover, in one of those individuals CTCs were shown to bear the $\mathrm{BRAF}^{\mathrm{V} 600 \mathrm{E}}$ mutation while this was not present at the tissue level, again suggesting that the CTCs reflect the heterogeneity of the tumors (Fusi et al., 2011). This is consistent with previous observations of intra- and inter-tumor heterogeneity of BRAF mutation status in melanoma (Sensi et al., 2006; Yancovitz et al., 2012). Inter- and intra-tumor heterogeneity have been identified in several tumor types and it has been shown to affect responses to targeted therapies in GIST and lung cancer (Liegl et al., 2008; Taniguchi et al., 2008). Given the diverse clinical responses of melanoma patients to BRAF inhibitors, studies on the association between tumor heterogeneity and clinical outcome are needed. In this context, CTCs could constitute an accessible sample with which to analyze the genetic profile of the tumors in a particular individual and possibly better represent the mutation status of all the tumors within a patient than a single biopsy.

Molecular characterization of CTCs for personalized treatment monitoring has been demonstrated in other tumors besides melanoma. For example Maheswaran et al. (2008) described a successful molecular analysis of CTCs from patients with metastatic non-small-cell lung cancer. The drug resistance mutation T790M was detected in CTCs collected from patients with EGFR mutations that had received tyrosine kinase inhibitors Gefitinib (Iressa) or Erlotinib (Tarceva). The presence of the mutation correlated with reduced PFS from 16.5 to 7.7 months $(p<0.001)$. This result supports the idea of monitoring changes in tumor genotypes during the course of treatment, by genotyping CTCs. Similarly, the presence of KRAS mutations in EGFR-positive colorectal cancer partially explains why these tumors do not respond to anti-EGFR mAb Cetuximab (Erbitux). Molecular analysis of the primary tumor determines the suitability of this targeted therapy, however discordances in the KRAS mutational status

\section{REFERENCES}

Anforth, R. M., Blumetti, T. C., Kefford, R. F., Sharma, R., Scolyer, R. A., Kossard, S., et al. (2012). Cutaneous manifestations of dabrafenib (GSK2118436): a selective inhibitor of mutant BRAF in patients with metastatic melanoma. Br. J. Dermatol. $167,1153-1160$.
Artale, S., Sartore-Bianchi, A., Veronese, S. M., Gambi, V., Sarnataro, C. S., Gambacorta, M., et al. (2008). Mutations of KRAS and BRAF in primary and matched metastatic sites of colorectal cancer. J. Clin. Oncol. 26, 4217-4219.

Attard, G., Swennenhuis, J. F., Olmos, D., Reid, A. H., Vickers, E., A'Hern, R.,

between the primary and metastatic tumors have been reported in a small subset of patients with metastatic colorectal cancer (Artale et al., 2008; Italiano et al., 2010). This could explain the observed resistance in some patients despite having a wildtype KRAS primary tumor. Yang et al. (2010) detected the KRAS mutation in blood CTCs and suggested that the blood might be a better sample to assess the tumor genotype for treatment decisions.

Chromosomal amplification of androgen receptor (AR), rearrangement of ERG gene, PTEN deletion, and MYC amplification were detected in CTCs from patients with metastatic prostate cancer by FISH (Attard et al., 2009; Leversha et al., 2009). Moreover, Attard and colleagues demonstrated that CTCs, metastases and prostate tissue invariably had the same ERG gene status in therapynaive prostate cancer patients. However, significant heterogeneity of AR copy number gain and PTEN loss were observed in CTCs, illustrating the heterogeneity of the tumors and the representation of this diversity in CTCs.

Altogether these observations support CTCs as a superior sample with which to examine the genetic profile of the sum of the patient's tumors and may therefore be useful for monitoring the development of escape mutations during treatment. Nevertheless, prior studies that isolate and analyze CTCs are limited in that they concentrate on methodologies that utilize only one or two surface proteins, gene deletions, amplifications, or point mutations. More comprehensive studies are required that determine the extent to which CTCs represent the parental tumors. The rapid progress in next generation sequencing and onco-proteomics will enable in the near future, better characterization of CTCs. Hopefully this will uncover more informative biomarkers with which to select CTCs and thus provide more specific information about patients who will benefit from targeted treatments as well as improve evaluation of therapeutic responses.

In parallel, improvements in the methodologies used to isolate and quantify CTCs are needed. Different methodologies that bias toward different tumor cell subsets might not reflect the overall tumor(s) heterogeneity. Issues such as collective migration (microemboli), epithelial-mesenchymal transition (EMT), and metastatic potential of the CTCs still need to be addressed in the context of well designed clinical trials with highly sensitive molecular analyses to determine which procedures provide the best prediction of clinical treatment outcomes. It is likely that this will be different for different cancer types and therapeutic interventions. The use of CTCs as a companion to treatments is a valuable tool that should be evaluated as part of therapy clinical trials to facilitate a swift implementation into clinical practice.

et al. (2009). Characterization of ERG, AR and PTEN gene status in circulating tumor cells from patients with castration-resistant prostate cancer. Cancer Res. 69, 2912-2918.

Berger, M. F., Hodis, E., Heffernan, T. P. Deribe, Y. L., Lawrence, M. S., Protopopov, A., et al. (2012). Melanoma genome sequencing reveals frequent PREX2 mutations. Nature 485, 502-506.

Bollag, G., Hirth, P., Tsai, J., Zhang, J., Ibrahim, P. N., Cho, H., et al. (2010). Clinical efficacy of a RAF inhibitor needs broad target blockade in BRAF-mutant melanoma. Nature 467, 596-599. 
Brahmer, J. R., Drake, C. G., Wollner, I., Powderly, J. D., Picus, J., Sharfman, W. H., et al. (2010). Phase I study of single-agent antiprogrammed death-1 (MDX-1106) in refractory solid tumors: safety, clinical activity, pharmacodynamics, and immunologic correlates. J. Clin. Oncol. 28, 3167-3175.

Brahmer, J. R., Tykodi, S. S., Chow, L. Q., Hwu, W. J., Topalian, S. L., Hwu, P., et al. (2012). Safety and activity of anti-PD-L1 antibody in patients with advanced cancer. N. Engl. J. Med. 366, 2455-2465.

Carvajal, R. D., Antonescu, C. R., Wolchok, J. D., Chapman, P. B., Roman, R. A., Teitcher, J., et al. (2011). KIT as a therapeutic target in metastatic melanoma. JAMA 305, 2327-2334.

Chapman, P. B., Hauschild, A., Robert, C., Haanen, J. B., Ascierto, P., Larkin, J., et al. (2011). Improved survival with Vemurafenib in melanoma with BRAF V600E mutation. N. Engl. J. Med. 364, 2507-2516.

Chapman, P. B., Hauschild, A., Robert, C., Larkin, J., Haanen, J. B., Ribas, A., et al. (2012). Updated overall survival (OS) results for BRIM-3, a phase III randomized, open-label, multicenter trial comparing BRAF inhibitor vemurafenib (vem) with dacarbazine (DTIC) in previously untreated patients with BRAFV600E-mutated melanoma. Melanoma/Skin Cancers 2012 ASCO Annual Meeting. J. Clin. Oncol. 30(Suppl.), abstr 8502.

Chappell, W. H., Steelman, L. S., Long, J. M., Kempf, R. C., Abrams, S. L., Franklin, R. A., et al. (2011). Ras/Raf/MEK/ERK and PI3K/PTEN/Akt/mTOR inhibitors: rationale and importance to inhibiting these pathways in human health. Oncotarget 2, 135-164.

Chow, L. Q., and Eckhardt, S. G. (2007). Sunitinib: from rational design to clinical efficacy. J. Clin. Oncol. 25, 884-896.

Cohen, S. J., Punt, C. J., Iannotti, N., Saidman, B. H., Sabbath, K. D., Gabrail, N. Y., et al. (2008). Relationship of circulating tumor cells to tumor response, progressionfree survival, and overall survival in patients with metastatic colorectal cancer. J. Clin. Oncol. 26, 3213-3221.

Colombino, M., Capone, M., Lissia, A., Cossu, A., Rubino, C., De Giorgi, V., et al. (2012). BRAF/NRAS mutation frequencies among primary tumors and metastases in patients with melanoma. J. Clin. Oncol. 30, 2522-2529.

Corcoran, R. B., Ebi, H., Turke, A. B., Coffee, E. M., Nishino, M., Cogdill,
A. P., et al. (2012). EGFR-mediated re-activation of MAPK signaling contributes to insensitivity of BRAF mutant colorectal cancers to RAF inhibition with vemurafenib. Cancer Discov. 2, 227-235.

Cristofanilli, M., Budd, G. T., Ellis, M. J., Stopeck, A., Matera, J., Miller, M. C., et al. (2004). Circulating tumor cells, disease progression, and survival in metastatic breast cancer. N. Engl. J. Med. 351, 781-791.

Curtin, J. A., Fridlyand, J., Kageshita, T., Patel, H. N., Busam, K. J., Kutzner, H., et al. (2005). Distinct sets of genetic alterations in melanoma. $N$. Engl. J. Med. 353, 2135-2147.

Davies, B. R., Logie, A., McKay, J. S., Martin, P., Steele, S., Jenkins, R., et al. (2007). AZD6244 (ARRY-142886), a potent inhibitor of mitogen-activated protein kinase/extracellular signalregulated kinase kinase 1/2 kinases: mechanism of action in vivo, pharmacokinetic/pharmacodynamic relationship, and potential for combination in preclinical models. Mol. Cancer Ther. 6, 2209-2219.

Davies, H., Bignell, G. R., Cox, C., Stephens, P., Edkins, S., Clegg, S., et al. (2002). Mutations of the BRAF gene in human cancer. Nature 417, 949-954.

de Bono, J. S., Scher, H. I., Montgomery, R. B., Parker, C., Miller, M. C., Tissing, H., et al. (2008). Circulating tumor cells predict survival benefit from treatment in metastatic castration-resistant prostate cancer. Clin. Cancer Res. 14, 6302-6309.

Deenen, M. J., Klumpen, H. J., Richel, D. J., Sparidans, R. W., Weterman, M. J., Beijnen, J. H., et al. (2012). Phase I and pharmacokinetic study of capecitabine and the oral mTOR inhibitor everolimus in patients with advanced solid malignancies. Invest. New Drugs 30, 1557-1565.

Di Giacomo, A. M., Ascierto, P. A., Pilla, L., Santinami, M., Ferrucci, P. F., Giannarelli, D., et al. (2012). Ipilimumab and fotemustine in patients with advanced melanoma (NIBIT-M1): an open-label, singlearm phase 2 trial. Lancet Oncol. 13, 879-886.

Dong, H., Zhu, G., Tamada, K., and Chen, L. (1999). B7-H1, a third member of the B7 family, costimulates T-cell proliferation and interleukin-10 secretion. Nat. Med. 5, 1365-1369.

Dong, J., Phelps, R. G., Qiao, R., Yao, S., Benard, O., Ronai, Z., et al. (2003). BRAF oncogenic mutations correlate with progression rather than initiation of human melanoma. Cancer Res. 63, 3883-3885.

Dronca, R. S., Perez, D. G., Allred, J., Maples, W. J., Creagan, E. T., Pockaj, B. A., et al. (2010). N0675: NCCTG phase II study of temozolomide (TMZ) and everolimus (RAD001) therapy for metastatic melanoma (MM). Melanoma/Skin Cancers 2010 ASCO Annual Meeting. J. Clin. Oncol. 28(Suppl. 15s), abstr 8572.

Dummer, R., Robert, C., Chapman, P. B., Sosman, J. A., Middleton, M. Bastholt, L., et al. (2008). AZD6244 (ARRY-142886) vs temozolomide (TMZ) in patients (pts) with advanced melanoma: an open-label, randomized, multicenter, phase II study. Melanoma/Skin Cancers 2008 ASCO Annual Meeting. J. Clin. Oncol. 26(Suppl.), abstr 9033.

Dutton-Regester, K., and Hayward, N. K. (2012). Reviewing the somatic genetics of melanoma: from current to future analytical approaches. Pigment Cell Melanoma Res. 25, 144154.

Eigentler, T. K., Figl, A., Krex, D., Mohr, P., Mauch, C., Rass, K., et al. (2011). Number of metastases, serum lactate dehydrogenase level, and type of treatment are prognostic factors in patients with brain metastases of malignant melanoma. Cancer 117, 1697-1703.

Eisen, T., Ahmad, T., Flaherty, K. T., Gore, M., Kaye, S., Marais, R., et al. (2006). Sorafenib in advanced melanoma: a Phase II randomised discontinuation trial analysis. $\mathrm{Br} . \mathrm{J}$. Cancer 95, 581-586.

Escudier, B., Eisen, T., Stadler, W. M., Szczylik, C., Oudard, S., Siebels, M., et al. (2007). Sorafenib in advanced clear-cell renal-cell carcinoma. $N$. Engl. J. Med. 356, 125-134.

Eskandarpour, M., Kiaii, S., Zhu, C. Castro, J., Sakko, A. J., and Hansson, J. (2005). Suppression of oncogenic NRAS by RNA interference induces apoptosis of human melanoma cells. Int. J. Cancer 115, 65-73.

Falchook, G. S., Lewis, K. D., Infante, J. R., Gordon, M. S., Vogelzang, N. J., Demarini, D. J., et al. (2012a). Activity of the oral MEK inhibitor trametinib in patients with advanced melanoma: a phase 1 doseescalation trial. Lancet Oncol. 13 782-789.

Falchook, G. S., Long, G. V., Kurzrock, R., Kim, K. B., Arkenau, T. H., Brown, M. P., et al. (2012b). Dabrafenib in patients with melanoma, untreated brain metastases, and other solid tumours: a phase 1 dose-escalation trial. Lancet 379, 1893-1901.
Fecher, L. A., Cummings, S. D., Keefe, M. J., and Alani, R. M. (2007) Toward a molecular classification of melanoma. J. Clin. Oncol. 25, 1606-1620.

Finn, R. S., Javie, B. R., Tan, J., Weekes, C. C., Bendell, J. C., Patnaik, A., et al. (2012). A phase I study of MEK inhibitor MEK162 (ARRY438162) in patients with biliary tract cancer. ASCO 2012 Gastrointestinal Cancers Symposium. J. Clin. Oncol. 30(Suppl. 4), abstr 220.

Flaherty, K. T. (2006). Chemotherapy and targeted therapy combinations in advanced melanoma. Clin. Cancer Res. 12, 2366s-2370s.

Flaherty, K. T., Infante, J. R., Daud, A., Gonzalez, R., Kefford, R. F., Sosman, J., et al. (2012a). Combined BRAF and MEK inhibition in melanoma with BRAF V600 mutations. N. Engl. J. Med. 367, 1694-1703.

Flaherty, K. T., Robert, C., Hersey, P., Nathan, P., Garbe, C., Milhem, M., et al. (2012b). Improved survival with MEK inhibition in BRAF-mutated melanoma. N. Engl. J. Med. 367 107-114.

Flores, J. F., Walker, G. J., Glendening, J. M., Haluska, F. G., Castresana, J. S. Rubio, M. P., et al. (1996). Loss of the p16INK4a and p15INK4b genes, as well as neighboring 9p21 markers, in sporadic melanoma. Cancer Res. 56, 5023-5032.

Fox, M. C., Lao, C. D., Schwartz, J. L., Frohm, M. L., Bichakjian, C. K., and Johnson, T. M. (2013). Management options for metastatic melanoma in the era of novel therapies: a primer for the practicing dermatologist: part II: management of stage IV disease. J. Am. Acad. Dermatol. 68 13.e11-e13.

Freeman, J. B., Gray, E. S., Millward, M., Pearce, R., and Ziman, M. (2012). Evaluation of a multimarker immunomagnetic enrichment assay for the quantification of circulating melanoma cells. J. Transl. Med. 10, 192.

Fusi, A., Berdel, R., Havemann, S., Nonnenmacher, A., and Keilholz, U. (2011). Enhanced detection of BRAF-mutants by pre-PCR cleavage of wild-type sequences revealed circulating melanoma cells heterogeneity. Eur. J. Cancer 47, 1971-1976.

Fusi, A., Liu, Z., Kummerlen, V., Nonnemacher, A., Jeske, J., and Keilholz, U. (2012). Expression of chemokine receptors on circulating tumor cells in patients with solid tumors. $J$. Transl. Med. 10, 52.

Gajewski, T. F., Niedzwiecki, D., Johnson, A. W., Linette, G., Bucher, C., Blaskovich, M., et al. (2006). Phase 
II study of the farnesyltransferase inhibitor R115777 in advanced melanoma: CALGB 500104. ASCO Annual Meeting Proceedings Part I. J. Clin. Oncol. 24(Suppl. 18S), abstr 8014.

Gartside, M. G., Chen, H., Ibrahimi, O. A., Byron, S. A., Curtis, A. V., Wellens, C. L., et al. (2009). Lossof-function fibroblast growth factor receptor-2 mutations in melanoma. Mol. Cancer Res. 7, 41-54.

Gray-Schopfer, V. C., Karasarides, M., Hayward, R., and Marais, R. (2007). Tumor necrosis factor-alpha blocks apoptosis in melanoma cells when BRAF signaling is inhibited. Cancer Res. 67, 122-129.

Guo, J., Si, L., Kong, Y., Flaherty, K. T., Xu, X., Zhu, Y., et al. (2011). Phase II, open-label, single-arm trial of imatinib mesylate in patients with metastatic melanoma harboring cKit mutation or amplification. $J$. Clin. Oncol. 29, 2904-2909.

Hamid, O. (2012). Efficacy and safety of MK-3475 in patients with advanced melanoma, Society for Melanoma Research 2012 Congress. Pigment Cell \& Melanoma Research 26, 150155.

Hauschild, A., Grob, J. J., Demidov, L. V., Jouary, T., Gutzmer, R., Millward, M., et al. (2012). Dabrafenib in BRAF-mutated metastatic melanoma: a multicentre, openlabel, phase 3 randomised controlled trial. Lancet 380, 358-365.

Hidalgo, M., and Rowinsky, E. K. (2000). The rapamycin-sensitive signal transduction pathway as a target for cancer therapy. Oncogene 19, 6680-6686.

Hodi, F. S., Friedlander, P., Corless, C. L., Heinrich, M. C., Mac Rae, S., Kruse, A., et al. (2008). Major response to imatinib mesylate in KIT-mutated melanoma. J. Clin. Oncol. 26, 2046-2051.

Hodi, F. S., O'day, S. J., McDermott, D. F., Weber, R. W., Sosman, J. A., Haanen, J. B., et al. (2010). Improved survival with ipilimumab in patients with metastatic melanoma. N. Engl. J. Med. 363, 711-723.

Hodis, E., Watson, I. R., Kryukov, G. V., Arold, S. T., Imielinski, M., Theurillat, J. P., et al. (2012). A landscape of driver mutations in melanoma. Cell $150,251-263$.

Hoshimoto, S., Faries, M. B., Morton, D. L., Shingai, T., Kuo, C., Wang, H. J., et al. (2012). Assessment of prognostic circulating tumor cells in a phase III trial of adjuvant immunotherapy after complete resection of stage IV melanoma. Ann. Surg. 255, 357-362.
Huang, S., and Houghton, P. J. (2003). Targeting mTOR signaling for cancer therapy. Curr. Opin. Pharmacol. 3, 371-377.

Italiano, A., Hostein, I., Soubeyran, I., Fabas, T., Benchimol, D., Evrard, S., et al. (2010). KRAS and BRAF mutational status in primary colorectal tumors and related metastatic sites: biological and clinical implications. Ann. Surg. Oncol. 17, 1429-1434.

Iwai, Y., Ishida, M., Tanaka, Y., Okazaki, T., Honjo, T., and Minato, N. (2002). Involvement of PD-L1 on tumor cells in the escape from host immune system and tumor immunotherapy by PD-L1 blockade. Proc. Natl. Acad. Sci. U.S.A. 99, 12293-12297.

Johannessen, C. M., Boehm, J. S., Kim, S. Y., Thomas, S. R., Wardwell, L., Johnson, L. A., et al. (2010). COT drives resistance to RAF inhibition through MAP kinase pathway reactivation. Nature 468, 968-972.

Kantarjian, H., Giles, F., Wunderle, L., Bhalla, K., O'Brien, S., Wassmann, B. et al. (2006). Nilotinib in imatinibresistant CML and Philadelphia chromosome-positive ALL. N. Engl. J. Med. 354, 2542-2551.

Khoja, L., Lorigan, P., Zhou, C., Lancashire, M., Booth, J., Cummings, J., et al. (2012). Biomarker utility of circulating tumor cells in metastatic cutaneous melanoma. J. Invest. Dermatol. doi:10.1038/jid.2012.468

Kim, K. B., Lewis, K., Pavlick, A., Infante, J. R., Ribas, A., Sosman, J. A., et al. (2011). A phase II study of the MEK1/MEK2 inhibitor GSK1 120212 in metastatic BRAF-V600E or $\mathrm{K}$ mutant cutaneous melanoma patients previously treated with or without a BRAF inhibitor. 2011 International Melanoma Congress. Pigment Cell Res. 24, 990-1075 (abstr; LBA1-3).

Kitago, M., Koyanagi, K., Nakamura, T., Goto, Y., Faries, M., O’Day, S. J., et al. (2009). mRNA expression and BRAF mutation in circulating melanoma cells isolated from peripheral blood with high molecular weight melanoma-associated antigenspecific monoclonal antibody beads. Clin. Chem. 55, 757-764.

Kluger, H. M., Dudek, A. Z., McCann, C., Ritacco, J., Southard, N., Jilaveanu, L. B., et al. (2011). A phase 2 trial of dasatinib in advanced melanoma. Cancer 117, 2202-2208.

Koyanagi, K., O’Day, S. J., Boasberg, P., Atkins, M. B., Wang, H. J., Gonzalez, R., et al. (2010). Serial monitoring of circulating tumor cells predicts outcome of induction biochemotherapy plus maintenance biotherapy for metastatic melanoma. Clin. Cancer Res. 16, 2402-2408.

Krauthammer, M., Kong, Y., Ha, B. H., Evans, P., Bacchiocchi, A., McCusker, J. P., et al. (2012). Exome sequencing identifies recurrent somatic RACl mutations in melanoma. Nat. Genet. 44, 1006-1014.

Krebs, M. G., Sloane, R., Priest, L., Lancashire, L., Hou, J. M., Greystoke, A., et al. (2011). Evaluation and prognostic significance of circulating tumor cells in patients with nonsmall-cell lung cancer. J. Clin. Oncol. 29, 1556-1563.

Lasithiotakis, K. G., Sinnberg, T. W. Schittek, B., Flaherty, K. T., Kulms, D., MacZey, E., et al. (2008). Combined inhibition of MAPK and mTOR signaling inhibits growth, induces cell death, and abrogates invasive growth of melanoma cells. J. Invest. Dermatol. 128, 2013-2023.

Leversha, M. A., Han, J., Asgari, Z., Danila, D. C., Lin, O., GonzalezEspinoza, R., et al. (2009). Fluorescence in situ hybridization analysis of circulating tumor cells in metastatic prostate cancer. Clin. Cancer Res. 15, 2091-2097.

Liegl, B., Kepten, I., Le, C., Zhu, M. Demetri, G. D., Heinrich, M. C., et al. (2008). Heterogeneity of kinase inhibitor resistance mechanisms in GIST. J. Pathol. 216, 64-74.

Linos, E., Swetter, S. M., Cockburn, M. G., Colditz, G. A., and Clarke, C. A. (2009). Increasing burden of melanoma in the United States. $J$. Invest. Dermatol. 129, 1666-1674.

Lo, R. S. (2012). Receptor tyrosine kinases in cancer escape from BRAF inhibitors. Cell Res. 22, 945-947.

Lombardo, L. J., Lee, F. Y., Chen, P., Norris, D., Barrish, J. C., Behnia, K., et al. (2004). Discovery of N-(2-chloro-6-methyl- phenyl)-2(6-(4-(2-hydroxyethyl)- piperazin1-yl)-2-methylpyrimidin-4ylamino) thiazole-5-carboxamide (BMS-354825), a dual Src/Abl kinase inhibitor with potent antitumor activity in preclinical assays. $J$. Med. Chem. 47, 6658-6661.

Long, G. V., Trefzer, U., Davies, M. A., Kefford, R. F., Ascierto, P. A., Chapman, P. B., et al. (2012). Dabrafenib in patients with Val600Glu or Val600Lys BRAF-mutant melanoma metastatic to the brain (BREAK$\mathrm{MB})$ : a multicentre, open-label, phase 2 trial. Lancet Oncol. 13, 1087-1095.

Lorusso, P. M., Adjei, A. A., Varterasian, M., Gadgeel, S., Reid, J., Mitchell, D. Y., et al. (2005). Phase I and pharmacodynamic study of the oral MEK inhibitor CI-1040 in patients with advanced malignancies. J. Clin. Oncol. 23, 5281-5293.

Lu, Y., Yu, Q., Liu, J. H., Zhang, J., Wang, H., Koul, D., et al. (2003). Src family protein-tyrosine kinases alter the function of PTEN to regulate phosphatidylinositol 3kinase/AKT cascades. J. Biol. Chem. 278, 40057-40066.

Maheswaran, S., Sequist, L. V., Nagrath, S., Ulkus, L., Brannigan, B., Collura, C. V., et al. (2008). Detection of mutations in EGFR in circulating lung-cancer cells. N. Engl. J. Med. 359, 366-377.

Manley, P. W., Stiefl, N., Cowan-Jacob, S. W., Kaufman, S., Mestan, J., Wartmann, M., et al. (2010). Structural resemblances and comparisons of the relative pharmacological properties of imatinib and nilotinib. Bioorg. Med. Chem. 18, 6977-6986.

Margolin, K., Longmate, J., Baratta, T., Synold, T., Christensen, S., Weber, J., et al. (2005). CCI-779 in metastatic melanoma: a phase II trial of the California Cancer Consortium. Cancer 104, 1045-1048.

Meier, F., Busch, S., Lasithiotakis, K., Kulms, D., Garbe, C., MacZey, E., et al. (2007). Combined targeting of MAPK and AKT signalling pathways is a promising strategy for melanoma treatment. Br. J. Dermatol. 156, 1204-1213.

Meier, F., Schittek, B., Busch, S., Garbe, C., Smalley, K., Satyamoorthy, K., et al. (2005). The RAS/RAF/MEK/ERK and PI3K/AKT signaling pathways present molecular targets for the effective treatment of advanced melanoma. Front. Biosci. 10, 2986-3001.

Menzies, A. M., Haydu, L. E., Visintin, L., Carlino, M. S., Howle, J. R., Thompson, J. F., et al. (2012). Distinguishing clinicopathologic features of patients with V600E and V600K BRAF-mutant metastatic melanoma. Clin. Cancer Res. 18, 3242-3249.

Minor, D. R., Kashani-Sabet, M., Garrido, M., O’Day, S. J., Hamid, O., and Bastian, B. C. (2012). Sunitinib therapy for melanoma patients with KIT mutations. Clin. Cancer Res. 18, 1457-1463.

Molhoek, K. R., Brautigan, D. L., and Slingluff, C. L. Jr. (2005). Synergistic inhibition of human melanoma proliferation by combination treatment with B-Raf inhibitor BAY43-9006 and mTOR inhibitor Rapamycin. $J$. Transl. Med. 3, 39.

Murugan, A. K., Dong, J., Xie, J., and Xing, M. (2009). MEK1 mutations, but not ERK2 mutations, occur in melanomas and colon carcinomas, 
but none in thyroid carcinomas. Cell Cycle 8, 2122-2124.

Nazarian, R., Shi, H., Wang, Q., Kong, X., Koya, R. C., Lee, H., et al. (2010). Melanomas acquire resistance to BRAF(V600E) inhibition by RTK or N-RAS upregulation. Nature 468, 973-977.

Nezos, A., Lembessis, P., Sourla, A., Pissimissis, N., Gogas, H., and Koutsilieris, M. (2009). Molecular markers detecting circulating melanoma cells by reverse transcription polymerase chain reaction: methodological pitfalls and clinical relevance. Clin. Chem. Lab. Med. 47, $1-11$.

Omholt, K., Krockel, D., Ringborg, U., and Hansson, J. (2006). Mutations of PIK3CA are rare in cutaneous melanoma. Melanoma Res. 16, 197-200.

Ott, P. A., Hamilton, A., Min, C., Safarzadeh-Amiri, S., Goldberg, L., Yoon, J., et al. (2010). A phase II trial of sorafenib in metastatic melanoma with tissue correlates. PLOS ONE 5:e15588. doi:10.1371/journal.pone.0015588

Paraiso, K. H., Xiang, Y., Rebecca, V. W., Abel, E. V., Chen, Y. A., Munko, A. C., et al. (2011). PTEN loss confers BRAF inhibitor resistance to melanoma cells through the suppression of BIM expression. Cancer Res. 71, 2750-2760.

Pavlu, J., and Marin, D. (2009). Dasatinib and chronic myeloid leukemia: two-year follow-up in eight clinical trials. Clin. Lymphoma Myeloma 9, 417-424.

Pecuchet, N., Lebbe, C., Mir, O., Billemont, B., Blanchet, B., Franck, N., et al. (2012). Sorafenib in advanced melanoma: a critical role for pharmacokinetics? Br. J. Cancer 107, 455-461.

Poulikakos, P. I., Persaud, Y., Janakiraman, M., Kong, X., Ng, C., Moriceau, G., et al. (2011). RAF inhibitor resistance is mediated by dimerization of aberrantly spliced BRAF(V600E). Nature 480, 387-390.

Prahallad, A., Sun, C., Huang, S., Di Nicolantonio, F., Salazar, R., Zecchin, D., et al. (2012). Unresponsiveness of colon cancer to BRAF(V600E) inhibition through feedback activation of EGFR. Nature 483, 100-103.

Prickett, T. D., Agrawal, N. S., Wei, X., Yates, K. E., Lin, J. C., Wunderlich, J. R., et al. (2009). Analysis of the tyrosine kinome in melanoma reveals recurrent mutations in ERBB4. Nat. Genet. 41, 1127-1132.

Prickett, T. D., Wei, X., Cardenas-Navia, I., Teer, J. K., Lin, J. C., Walia, V., et al. (2011). Exon capture analysis of $\mathrm{G}$ protein-coupled receptors identifies activating mutations in GRM3 in melanoma. Nat. Genet. 43, 1119-1126.

Reid, A. L., Millward, M., Pearce, R., Lee, M., Frank, M. H., Ireland, A., et al. (2013). Markers of circulating tumour cells in the peripheral blood of patients with melanoma correlate with disease recurrence and progression. Br. J. Dermatol. 168, 85-92.

Reynolds, S. R., Albrecht, J., Shapiro, R. L., Roses, D. F., Harris, M. N., Conrad, A., et al. (2003). Changes in the presence of multiple markers of circulating melanoma cells correlate with clinical outcome in patients with melanoma. Clin. Cancer Res. 9, 1497-1502.

Ribas, A. (2012). Tumor immunotherapy directed at PD-1. N. Engl. J. Med. 366, 2517-2519.

Robert, C., Flaherty, K. T., Hersey, P., Nathan, P., Garbe, C., Milhem, M. M., et al. (2012). METRIC phase III study: efficacy of trametinib (T), a potent and selective MEK inhibitor (MEKi), in progression-free survival (PFS) and overall survival (OS), compared with chemotherapy (C) in patients (pts) with BRAFV600E/K mutant advanced or metastatic melanoma (MM). Melanoma/Skin Cancers 2012 ASCO Annual Meeting. J. Clin. Oncol. 30(Suppl.), abstr LBA8509.

Robert, C., Thomas, L., Bondarenko, I., O'Day, S. M. D. J., Garbe, C., Lebbe, C., et al. (2011). Ipilimumab plus dacarbazine for previously untreated metastatic melanoma. $N$. Engl. J. Med. 364, 2517-2526.

Roberts, P. J., and Der, C. J. (2007). Targeting the Raf-MEK-ERK mitogenactivated protein kinase cascade for the treatment of cancer. Oncogene 26, 3291-3310.

Sakaizawa, K., Goto, Y., Kiniwa, Y., Uchiyama, A., Harada, K., Shimada, S., et al. (2012). Mutation analysis of BRAF and KIT in circulating melanoma cells at the single cell level. Br. J. Cancer 106, 939-946.

Schwartz, G. K., Robertson, S., Shen, A., Wang, E., Pace, L., Dials, H., et al. (2009). A phase I study of XL281, a selective oral RAF kinase inhibitor, in patients (Pts) with advanced solid tumors. Melanoma/Skin Cancers 2009 ASCO Annual Meeting. J. Clin. Oncol. 27(Suppl. 15s), abstr 3513.

Sebti, S. M. (2005). Protein farnesylation: implications for normal physiology, malignant transformation, and cancer therapy. Cancer Cell 7, 297-300.

Sensi, M., Nicolini, G., Petti, C., Bersani, I., Lozupone, F., Molla, A., et al. (2006). Mutually exclusive NRASQ61R and BRAFV600E mutations at the single-cell level in the same human melanoma. Oncogene $25,3357-3364$.

Shi, H., Kong, X., Ribas, A., and Lo, R. S. (2011). Combinatorial treatments that overcome PDGFRbeta-driven resistance of melanoma cells to V600EBRAF inhibition. Cancer Res. 71, 5067-5074.

Shi, H., Moriceau, G., Kong, X., Koya, R. C., Nazarian, R., Pupo, G. M., et al. (2012a). Preexisting MEK1 exon 3 mutations in V600E/KBRAF melanomas do not confer resistance to BRAF inhibitors. Cancer Discov. 2 , 414-424.

Shi, H., Moriceau, G., Kong, X., Lee, M.-K., Lee, H., Koya, R. C., et al. (2012b). Melanoma whole-exome sequencing identifies V600EB-RAF amplification-mediated acquired BRAF inhibitor resistance. Nat. Commun. 3, 724 .

Sosman, J. A., Kim, K. B., Schuchter, L., Gonzalez, R., Pavlick, A. C. Weber, J. S., et al. (2012). Survival in BRAF V600-mutant advanced melanoma treated with vemurafenib. N. Engl. J. Med. 366, 707-714.

Stark, M. S., Woods, S. L., Gartside, M. G., Bonazzi, V. F., DuttonRegester, K., Aoude, L. G., et al. (2012). Frequent somatic mutations in MAP3K5 and MAP3K9 in metastatic melanoma identified by exome sequencing. Nat. Genet. 44, 165-169.

Stuart, D., and Sellers, W. R. (2009). Linking somatic genetic alterations in cancer to therapeutics. Curr. Opin. Cell Biol. 21, 304-310.

Su, F., Viros, A., Milagre, C., Trunzer, K., Bollag, G., Spleiss, O., et al. (2012a). RAS mutations in cutaneous squamous-cell carcinomas in patients treated with BRAF inhibitors. N. Engl. J. Med. 366, 207-215.

Su, Y., Vilgelm, A. E., Kelley, M. C., Hawkins, O. E., Liu, Y., Boyd, K. L., et al. (2012b). RAF265 inhibits the growth of advanced human melanoma tumors. Clin. Cancer Res. 18, 2184-2198.

Taniguchi, K., Okami, J., Kodama, K., Higashiyama, M., and Kato, K. (2008). Intratumor heterogeneity of epidermal growth factor receptor mutations in lung cancer and its correlation to the response to gefitinib. Cancer Sci. 99, 929-935.

Topalian, S. L., Hodi, F. S., Brahmer, J. R., Gettinger, S. N., Smith, D. C., McDermott, D. F., et al. (2012). Safety, activity, and immune correlates of anti-PD-1 antibody in cancer. N. Engl. J. Med. 366, 2443-2454.

Ugurel, S., Hildenbrand, R., Zimpfer, A., La Rosee, P., Paschka, P., Sucker, A., et al. (2005). Lack of clinical efficacy of imatinib in metastatic melanoma. Br. J. Cancer 92, 1398-1405.

US Food and Drug Administration. (2011). FDA labelling information Zelboraf. Available at: http://www. accessdata.fda.gov/drugsatfda docs/label/2011/202429s000lbl.pdf

Van Raamsdonk, C. D., Griewank, K. G., Crosby, M. B., Garrido, M. C., Vemula, S., Wiesner, T., et al. (2010). Mutations in GNA11 in uveal melanoma. N. Engl. J. Med. 363, 2191-2199.

Venetsanakos, E., Stuart, D., Tan, N., Ye, H., Salangsang, F., Aardalen, K. et al. (2006). CHIR-265, a novel inhibitor that targets B-Raf and VEGFR, shows efficacy in a broad range of preclinical models. Proc. Amer. Assoc. Cancer Res. 47, abstr 4854.

Villanueva, J., Vultur, A., Lee, J. T., Somasundaram, R., FukunagaKalabis, M., Cipolla, A. K., et al. (2010). Acquired resistance to BRAF inhibitors mediated by a RAF kinase switch in melanoma can be overcome by cotargeting MEK and IGF-1R/PI3K. Cancer Cell 18, 683-695.

von Mehren, M. (2006). Beyond imatinib: second generation c-KIT inhibitors for the management of gastrointestinal stromal tumors. Clin. Colorectal Cancer 6(Suppl. 1), S30-S34.

Wagle, N., Emery, C., Berger, M. F., Davis, M. J., Sawyer, A., Pochanard, P., et al. (2011). Dissecting therapeutic resistance to RAF inhibition in melanoma by tumor genomic profiling. J. Clin. Oncol. 29, 3085-3096.

Webster, J. D., Kiupel, M., and Yuzbasiyan-Gurkan, V. (2006). Evaluation of the kinase domain of c-KIT in canine cutaneous mast cell tumors. BMC Cancer 6:85. doi:10.1186/1471-2407-6-85

Wei, X., Walia, V., Lin, J. C., Teer, J. K., Prickett, T. D., Gartner, J., et al. (2011). Exome sequencing identifies GRIN2A as frequently mutated in melanoma. Nat. Genet. 43, 442-446. Wellbrock, C., Karasarides, M., and Marais, R. (2004). The RAF proteins 
take centre stage. Nat. Rev. Mol. Cell Biol. 5, 875-885.

Wilhelm, S., Carter, C., Lynch, M., Lowinger, T., Dumas, J., Smith, R. A., et al. (2006). Discovery and development of sorafenib: a multikinase inhibitor for treating cancer. Nat. Rev. Drug Discov. 5, 835-844.

Wilmott, J. S., Long, G. V., Howle, J. R., Haydu, L. E., Sharma, R. N., Thompson, J. F., et al. (2012). Selective BRAF inhibitors induce marked T-cell infiltration into human metastatic melanoma. Clin. Cancer Res. 18, 1386-1394.

Wyman, K., Atkins, M. B., Prieto, V., Eton, O., McDermott, D. F., Hubbard, F., et al. (2006). Multicenter Phase II trial of high-dose imatinib mesylate in metastatic melanoma: significant toxicity with no clinical efficacy. Cancer 106, 2005-2011.

Yancovitz, M., Litterman, A., Yoon, J., Ng, E., Shapiro, R. L., Berman, R. S., et al. (2012). Intra- and inter-tumor heterogeneity of $\mathrm{BRAF}(\mathrm{V} 600 \mathrm{E})$ mutations in primary and metastatic melanoma. PLoS ONE 7:e29336. doi:10.1371/journal.pone.0029336

Yang, M. J., Chiu, H. H., Wang, H. M., Yen, L. C., Tsao, D. A., Hsiao, C. P., et al. (2010). Enhancing detection of circulating tumor cells with activating KRAS oncogene in patients with colorectal cancer by weighted chemiluminescent membrane array method. Ann. Surg. Oncol. 17, 624-633.
Yeh, T. C., Marsh, V., Bernat, B. A., Ballard, J., Colwell, H., Evans, R. J., et al. (2007). Biological characterization of ARRY-142886 (AZD6244), a potent, highly selective mitogen-activated protein kinase kinase $1 / 2$ inhibitor. Clin. Cancer Res. 13, 1576-1583.

Conflict of Interest Statement: The authors declare that the research was conducted in the absence of any commercial or financial relationships that could be construed as a potential conflict of interest.

Received: 17 December 2012; accepted: 28 February 2013; published online: 19 March 2013.
Citation: Klinac D, Gray ES, Millward $M$ and Ziman M (2013) Advances in personalized targeted treatment of metastatic melanoma and non-invasive tumor monitoring. Front. Oncol. 3:54. doi: 10.3389/fonc.2013.00054

This article was submitted to Frontiers in Cancer Genetics, a specialty of Frontiers in Oncology.

Copyright (c) 2013 Klinac, Gray, Millward and Ziman. This is an openaccess article distributed under the terms of the Creative Commons Attribution License, which permits use, distribution and reproduction in other forums, provided the original authors and source are credited and subject to any copyright notices concerning any third-party graphics etc. 